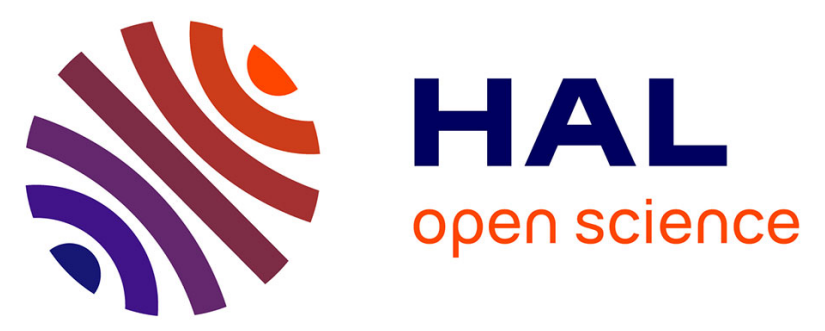

\title{
The interaction between flow-induced vibration mechanisms of a square cylinder with varying angles of attack
}

Andras Nemes, Jisheng Zhao, David Lo Jacono, John Sheridan

\section{- To cite this version:}

Andras Nemes, Jisheng Zhao, David Lo Jacono, John Sheridan. The interaction between flow-induced vibration mechanisms of a square cylinder with varying angles of attack. Journal of Fluid Mechanics, 2012, vol. 710, pp. 102-130. 10.1017/jfm.2012.353 . hal-00752464

\author{
HAL Id: hal-00752464 \\ https://hal.science/hal-00752464
}

Submitted on 15 Nov 2012

HAL is a multi-disciplinary open access archive for the deposit and dissemination of scientific research documents, whether they are published or not. The documents may come from teaching and research institutions in France or abroad, or from public or private research centers.
L'archive ouverte pluridisciplinaire HAL, est destinée au dépôt et à la diffusion de documents scientifiques de niveau recherche, publiés ou non, émanant des établissements d'enseignement et de recherche français ou étrangers, des laboratoires publics ou privés. 


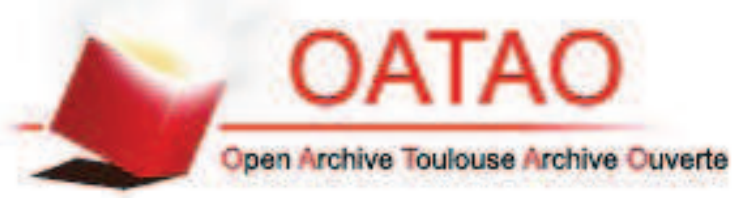

\section{Open Archive TOULOUSE Archive Ouverte (OATAO)}

OATAO is an open access repository that collects the work of Toulouse researchers and makes it freely available over the web where possible.

This is an author-deposited version published in : http://oatao.univ-toulouse.fr/ Eprints ID : 6867

To link to this article : DOI: $10.1017 / \mathrm{jfm} .2012 .353$

URL : http://dx.doi.org/10.1017/jfm.2012.353

To cite this version : Nemes, Andras and Zhao, Jisheng and Lo Jacono, David and Sheridan, John The interaction between flowinduced vibration mechanisms of a square cylinder with varying angles of attack. (2012) Journal of Fluid Mechanics, vol. 710 . pp. 102-130. ISSN 0022-1120

Any correspondence concerning this service should be sent to the repository administrator: staff-oatao@listes.diff.inp-toulouse.fr 


\title{
The interaction between flow-induced vibration mechanisms of a square cylinder with varying angles of attack
}

\author{
András Nemes $^{1} \dagger$, Jisheng Zhao ${ }^{1}$, David Lo Jacono ${ }^{1,2,3}$ and John Sheridan ${ }^{1}$ \\ ${ }^{1}$ Fluids Laboratory for Aeronautical and Industrial Research (FLAIR), Department of Mechanical and \\ Aerospace Engineering, Monash University, Melbourne, Victoria 3800, Australia \\ 2 Université de Toulouse, INPT, UPS, IMFT (Institut de Mécanique des Fluides de Toulouse), \\ Allée Camille Soula, F-31400 Toulouse, France \\ ${ }^{3}$ CNRS, IMFT, F-31400 Toulouse, France
}

This study examines the influence of angle of attack of a square section cylinder on the cylinder's flow-induced vibration, where the direction of the vibration is transverse to the oncoming flow. Our experiments, which traversed the velocity-angle of attack parameter space in considerable breadth and depth, show that a low-mass ratio body can undergo combinations of both vortex-induced vibration and galloping. When the body has an angle of attack that makes it symmetric to the flow, such as when it assumes the square or diamond orientation, the two mechanisms remain independent. However, when symmetry is lost we find a mixed mode response with a new branch of vortex-induced oscillations that exceeds the amplitudes resulting from the two phenomena independently. The oscillations of this higher branch have amplitudes larger than the 'upper branch' of vortex-induced vibrations and at half the frequency. For velocities above this resonant region, the frequency splits into two diverging branches. Analysis of the amplitude response reveals that the transition between galloping and vortex-induced vibrations occurs over a narrow range of angle of incidence. Despite the rich set of states found in the parameter space the vortex shedding modes remain very similar to those found previously in vortex-induced vibration.

Key words: vortex shedding, wakes, wake-structure interactions

\section{Introduction}

Flow-induced vibration (FIV) of bluff bodies has received considerable attention in the last four decades. Its prevalence and importance in practical engineering applications has motivated investigations that aim to enable us to predict, model and shed light on these fluid-structure interactions. This has led to a large body of work, well covered in collections by Blevins (1990) and Naudascher \& Rockwell (1994). Two body-oscillator phenomena in particular, vortex-induced vibration (VIV) and galloping, have been researched extensively for their ubiquity in nature and their serious implications in wind and ocean engineering. 
VIV has been the subject of many applied and fundamental studies. The focus in the field has been on bodies with circular cross-sections, a canonical bluff body with an axial symmetry that removes any parametric consideration relating to the flow orientation, that serves as an ideal basis for fundamental research. This symmetry also allows VIV to be studied in isolation from other FIV, such as galloping, that arise from aerodynamically unstable cross-sections (Blevins 1990). From an applied standpoint, VIV of the circular cross-section receives considerable attention as a critical phenomena in ocean engineering, where ocean currents can induce vibrations in ocean risers, cables and off-shore platforms. This requires prediction and accounting for the resulting cyclic loading and maximal displacements induced in structures.

VIV can occur when an elastic or elastically mounted bluff body immersed in a moving fluid sheds vortices. These vortices create a fluctuating pressure distribution on the body that may induce a vibrational response at certain velocities. Here we focus on the VIV response of a single degree of freedom system with oscillations transverse to the direction of the fluid flow.

The dynamics of this response are defined by the equation

$$
m \ddot{y}+c \dot{y}+k y=F_{y}(t),
$$

where $m$ is the mass of the system, $c$ is the structural damping, $k$ is the spring constant, $y$ is the cylinder transverse displacement and $F_{y}(t)$ is the forcing imposed on the cylinder by the fluid. This leads to the key non-dimensional parameters of the system: the mass ratio, $m^{*}=m / m_{d}$, where $m_{d}$ is the mass of the fluid displaced by the body; and the damping ratio of the system in water,

$$
\zeta=c /\left(2 \sqrt{k\left(m+m_{A}\right)}\right),
$$

in which $m_{A}$ is the added mass. The displaced fluid mass is defined as $m_{d}=\rho C L$, where $\rho$ is the fluid density, $C$ is the geometry's cross-sectional area, and $L$ is the length of the body immersed in the fluid. A pioneering wind tunnel experiment by Feng (1968) characterized the vibrations of a high mass ratio circular cylinders by the oscillation amplitude and frequency response of the body over a range of reduced velocities, $U^{*}=U / f_{N} D$, where $U$ is the free stream velocity, $f_{N}$ is the natural frequency of the freely oscillating body within the quiescent fluid and $D$ is the cylinder diameter Feng showed that a resonance condition can exist when the frequency of shedding, $f_{S}$, and that of the body oscillation, $f$, are locked and close to $f_{N}$. The maximum amplitude response, $A_{\max }^{*}=\max (A / D)$, occurs in this lock-in region of $f_{S} \approx f \approx f_{N}$, i.e. after the reduced velocity increases above, $U^{*}=1 / S t$, where $S t=f_{S} D / U$ is the fixed cylinder's dimensionless shedding frequency.

The amplitude envelope found by Feng (1968) was a two-branch response with a hysteretic transition and a maximum amplitude of just over half the body diameter, $A_{\max }^{*}=0.5$. Since Feng (1968), a great deal of work has been done on VIV, with experimental and numerical studies that have covered the large number of parameters involved. To understand, model and predict the complex coupling of the fluid-structure interaction, researchers have characterized the response of circular cylinders undergoing free and forced vibrations with multiple degrees of freedom and over a large range of parameters. The state of the art has been well documented and covered in reviews by Sarpkaya (1979, 2004), Bearman (1984), Parkinson (1989) and Williamson \& Govardhan (2004).

Khalak \& Williamson $(1996,1997 a, b, 1999)$ conducted a series of one-degreeof-freedom circular cylinder experiments in a water channel. They showed a larger 
vibrational amplitude response than Feng's (1968) could result when the mass-damping values, $m^{*} \zeta$, were very low. They also found that this extended the synchronization region to higher $U^{*}$ values and that within this extended region $f^{*}=f / f_{N}$ could take values above unity. These studies, and subsequent low mass-damping experiments (Govardhan \& Williamson 2000, 2004), revealed that three distinct branches of maximum amplitude can occur within the resonance region. As the flow velocity is increased from low values the body oscillates at low amplitude, coined the 'initial branch'. At $U^{*}=1 / S t$ there is a hysteretic jump to an 'upper branch' of highamplitude oscillations, which can exceed $A_{\max }^{*}=1$. Govardhan \& Williamson (2006) found the magnitude of the peak amplitude is influenced not just by mass-damping but also by Reynolds number, $R e=U D / v$, where $v$ is the kinematic viscosity of the fluid.

With a further increase in $U^{*}$ there is a drop in amplitude to a 'lower branch' of highly periodic oscillations, with the transition between the 'upper' and 'lower' branches being found to be intermittent (Khalak \& Williamson 1997a). Throughout the velocity range of the lower branch the body motion and lift remain locked to a constant $f^{*}$ value, above unity and inversely proportional to $m^{*}$ (Govardhan \& Williamson 2004). Near the end of the lower branch, the frequencies desynchronize and the oscillations of the body drop to a low amplitude. This drop was also shown to be a hysteretic transition by Klamo, Leonard \& Roshko (2006). They showed that the existence of the three-branch response depends on having both high Reynolds numbers and low mass-damping.

There has been little research into pure VIV of square cross-section cylinders, probably because the circular cylinder serves as an ideal geometry isolated from other FIV phenomena. Obasaju, Ermshaus \& Naudascher (1990) studied flow over square section cylinders at different angles of incidence while freely oscillating in the streamwise direction, reporting on amplitude-dependent vortex shedding modes and multiple FIV sources of excitation. Wang \& Zhou (2005) located the resonance modes of an elastic square cylinder with fixed supports over an extensive velocity range. Recently, there has been a renewed interest in studying the flow around stationary square sections at angles of incidence experimentally (see Dutta, Panigrahi \& Muralidhar 2008; van Oudheusden et al. 2008) and numerically (see Luo, Tong \& Khoo 2007; Tong, Luo \& Khoo 2008; Sheard, Fitzgerald \& Ryan 2009; Yoon, Yang \& Choi 2010). These studies have focused on the fluid forces, reattachment and wake modes, furthering the early work of Vickery (1966), Okajima (1982) and Norberg (1993). The consideration of wind direction over buildings, bridges and other rectangular structures is particularly important in civil engineering, as it leads to variation in the structure's angle of attack. This may result in shear layer reattachment and uneven pressure distributions that can lead to unpredictable loading patterns. This has led to a recognized need to better understand the FIV of such structures at varying angles of incidence.

The second mode of FIV, galloping, is an aerodynamic instability experienced by bodies with non-axisymmetric cross-sections that has been extensively studied since Den Hartog (1932) first proposed his criterion for galloping of ice-covered cables. It is characterized by low-frequency oscillations that increase in amplitude unbounded with fluid velocity. The galloping is driven by the instantaneous angle of attack between the body and the flow, $\beta=\tan ^{-1}\left(\dot{y} / U_{\infty}\right)$, producing an asymmetric pressure distribution. Any asymmetric slender body in a cross-flow is susceptible to galloping at certain angles, however flow over square section cylinders has been studied most frequently due in part to the loss of only one degree of symmetry compared with the circular cylinder and the simplest geometry that can gallop. Parkinson \& Smith 
(1964) provided the foundations for the current state of the art with a model for the galloping response of a square section at zero angle of incidence using a quasisteady formulation of stationary cylinder lift force measurements. Quasi-steady theory accurately predicts the amplitude response of a square section, including the critical velocity required for the onset of galloping and a velocity dependent hysteresis in the amplitude response.

Irrespective of their orientation, square cross-section cylinders have fixed separation points and a region of the body immersed in the separated wake, the afterbody, making the geometry susceptible to both VIV and galloping. Parkinson \& Wawzonek (1981) showed that the onset of galloping of rectangular cross-sections can lie in the range of velocities at which a body can also experience VIV, with the possibility that the two phenomena could occur simultaneously. This raises the question whether the combination of the two phenomena produces larger vibrations than produced by either model independently. Bokaian \& Geoola (1984) observed experimentally a combination of galloping and VIV, leading to a modified quasi-steady theory to account for the combined effects. Further experiments by Bearman et al. (1987) on the mixed mode of galloping and VIV of a square cross-section revealed surface pressure frequencies at both the stationary shedding frequency and the oscillation frequency. For low damping values they concluded that the complex interaction could not be explained by the individual models. Corless \& Parkinson $(1988,1993)$ used the Hartlin-Currie VIV wake-oscillator model and the quasi-steady theory to model the combined effects with multiple time scales, the results agreed reasonably well with the experiments of Bearman et al. (1987). All of these studies were on oscillating square cylinders with $\alpha=0^{\circ}$, the square orientation. Naudascher \& Wang (1993) have reviewed the effect of angle of incidence on flow over prismatic structures but this was not extended to the study of the transition between VIV and galloping or their combined effect. Deniz \& Staubli (1997) performed forced experiments on octagonal (length/width $=3.33$ ) and rectangular cross-sections (length/width $=2)$ at two angles of attack $\left(\alpha=0\right.$ and $\left.10^{\circ}\right)$ and studied the interaction effects of VIV and galloping at high Reynolds numbers $(R e>64000)$.

The present study investigates the influence of the angle of attack of a freely vibrating square cylinder and the response modes that result. The cylinder used has a low-mass-damping ratio, this being chosen because it produces a high-amplitude response. The angle of attack was varied in small increments from 0 to $45^{\circ}$, i.e. the square to diamond configurations. In this range we would expect to see both galloping and VIV; our focus here is on the regions in which each dominates, the transition between them and modes arising from their combination.

The experimental method, apparatus and acquisition procedures are detailed in the following section. Section 3 provides a brief validation of the experimental apparatus and method, followed by a discussion of the results of the free oscillations in $\S \S 4$ and 5 in the limiting case of the diamond and square configuration respectively. Section 6 presents the body's response over a parameter map of reduced velocity and angle of attack. Here there is a focus on a new higher-amplitude branch of oscillation found within a mixed mode region of parameter space that is highly dependent on both velocity and angle of attack. This parameter map is proposed as a good basis for validation of numerical studies, and complemented by discussion on the amplitude and frequency response with varying angle of incidence. 


\section{Experimental method}

\subsection{Experimental apparatus}

The experiments were conducted in the free-surface recirculating water channel of FLAIR in the Department of Mechanical and Aerospace Engineering at Monash University. The water channel facility has a test section of $4000 \mathrm{~mm}$ in length, $600 \mathrm{~mm}$ in width and $800 \mathrm{~mm}$ in depth.

Two bluff body models were used in the present study: a square cross-section rigid cylinder model made from aluminium tubing with a side width of $W=25 \mathrm{~mm}$ and wall thickness of $1.6 \mathrm{~mm}$; and a carbon fibre circular cylinder of diameter $D=25 \mathrm{~mm}$ and wall thickness of $1.5 \mathrm{~mm}$. Both cylinders contained a $10 \mathrm{~mm}$ long Perspex crosssection $200 \mathrm{~mm}$ from the end to allow optical access. The models had an immersed length of $778 \mathrm{~mm}$, both with an aspect ratio of $A R=31$ (for the square cylinder this was calculated based on side length) leaving a $2 \mathrm{~mm}$ gap between the floor of the channel and the cylinders to minimize end effects. Choice and influence of this end condition are discussed in $\S 3$. The cylinders were vertically supported via a sting by a pair of carbon fibre shafts mounted on air bearing bushings (Porous Air Bushings S302502, New Way Air Bearings, USA), and elastically constrained by a pair of extension springs (LE014B13S, Lee Spring), allowing the cylinder one degree of freedom in the transverse direction to the oncoming flow. The system had a total stroke length of $160 \mathrm{~mm}$. The total mass of the system undergoing motion for the square cross-section cylinder was $m=1065 \mathrm{~g}$ and the mass of the displaced water was $m_{d}=483 \mathrm{~g}$, resulting in a mass ratio close to $m^{*}=2.2$. The circular cylinder total mass was $m=836 \mathrm{~g}$, which resulted in the same mass ratio. The natural frequency in water, $f_{N}$, was measured with free decay tests taken in quiescent water. The natural frequency in air, $f_{N a}$, was measured from air free decay tests, along with the structural damping, $c$. The damping in water, $\zeta$, was then calculated from (1.2). This was done with the cylinders removed and their equivalent weights added to the air bearing rig, which removed the effect of the fluid-structure interaction of the air and cylinder. The damping ratio of the system was $\zeta=2.95 \times 10^{-3}$, giving a mass-damping ratio of $m^{*} \zeta=6.49 \times 10^{-3}$.

\subsection{Measurements}

Particle image velocimetry (PIV) was used to determine the vortex structures that arise from FIV in the near wake of the cylinders. The flow was seeded with hollow microspheres (Sphericel 110P8, Potters Industries Inc.) with a nominal diameter of $13 \mu \mathrm{m}$ and a specific weight of $1.1 \mathrm{~g} \mathrm{~cm}^{-3}$. Two miniature Nd:YAG pulsed lasers (Minilite II Q-Switched lasers, Continuum) produced a $2 \mathrm{~mm}$ thick horizontal planar sheet, illuminating the particles in the plane of the body cross-section. A CCD camera of $2048 \times 2048$ pixels (PCO 2000, PCO AG), equipped with a $50 \mathrm{~mm}$ lens (Nikkor, Nikon Corporation), captured the PIV image pairs. The data were analysed with in-house PIV software (Fouras, Lo Jacono \& Hourigan 2008), using $32 \times 32$ pixels interrogation windows in a grid with $50 \%$ window overlap. The laser's TTL trigger signal and the position and force measurements were sampled at $100 \mathrm{~Hz}$ by a data acquisition system (BNC-2110 Connector Block attached to NI PCI-6221 DAQ Board) allowing the captured velocity and vorticity fields to be phase-averaged based on the cylinder position. At least 1000 image pairs were taken at each velocity represented, resulting in over 100 image pairs averaged at each of the eight phases of the oscillation cycle.

A non-contact magnetostrictive linear variable differential transformer (SE 750-10000, Macro Sensors) accurately measured the displacement of the cylinder to 


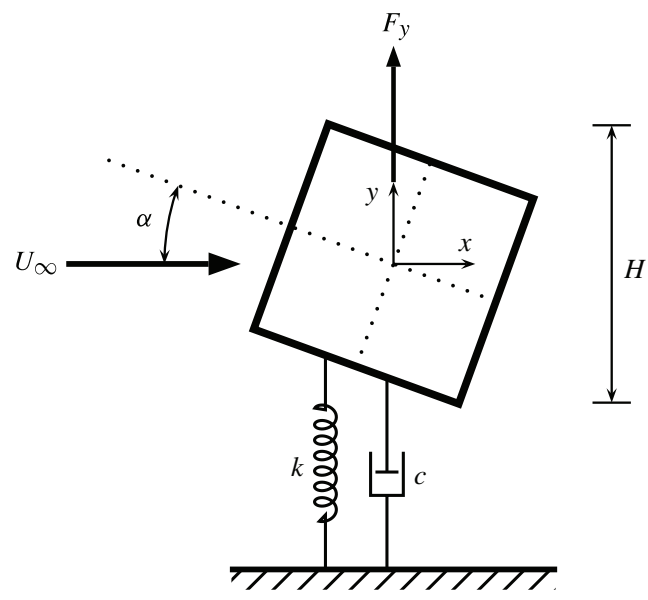

FIGURE 1. Schematic of the experimental rig showing the square cylinder with variable angle of attack, $\alpha$, and projected length, $H$, freely oscillating in the transverse direction, $y$, to the oncoming flow, $U_{\infty}$ in the stream-wise direction $x$. The body is mounted and elastically constrained to provide $k$, the system spring constant, and $c$, the structural damping.

within $\pm 0.01 \%$ of the $250 \mathrm{~mm}$ linear range available, for a displacement precision of $0.001 \mathrm{H}$. The lift and drag forces acting on the cylinder were recorded with two-component force and moment strain gauges in Wheatstone bridge configurations mounted on a sting above the cylinder. Forces were calculated from the moment strain gauges, having larger signal-to-noise ratio (SNR) in the voltage measurements. For the lift force calculation, the inertial force of the bodies' motions were taken into account to recover the instantaneous fluid force, $F(t)$, acting on a body. To obtain a reference the zero position of the cylinder was measured for each angle in a quiescent fluid. The force balance gauges were calibrated in the same way.

Experimental results were recorded over a $20 \mathrm{~min}$ period for each parameter combination of velocity and angle of attack. This provided accurate representation of the phenomena for a minimum of 800 oscillations and was found to be long enough to successfully capture the intermittent behaviour in the transitional regimes discussed in $\S 6.4$.

Frequencies for the periodic position and lift time histories were extracted using fast-Fourier transforms (FFTs) and continuous wavelet transforms (CWTs). Morlet wavelets were used as the mother wavelet in the CWTs over a time-bandwidth spanning the entire run duration and a frequency range of $0.0128-4 \mathrm{~Hz}$. This range encompassed the relevant frequencies of interest encountered in both the stationary and freely vibrating cylinder experiments. Frequency peaks for acquisition periods were determined using temporally averaged power spectra from the CWTs and confirmed by the FFTs. Peak detection with thresholding was used to extract the frequencies of the maxima of the averaged power spectra from the wavelet analysis. The phase, $\phi_{t}$, between body position and lift force was determined using cross-wavelet transforms (XWTs). This provided the temporal instantaneous phase between the signals and the shared spectral power over the entire frequency-time bandwidth. The instantaneous phase relationship at the frequency of the dominant oscillation was sorted by phase angle and the mode of the distribution, the angle with the most samples, was used as the total phase, $\phi_{t}$, at each flow velocity and angle combination. 


\section{Vortex-induced vibration of a circular cylinder}

Prior to discussing the response of the diamond cylinder, it is useful to first validate our apparatus and experimental approach using the well-studied geometry of a circular cylinder.

We conducted a series of validation experiments using circular cylinders with three different end-conditions to ascertain their influence on the amplitude response. Morse, Govardhan \& Williamson (2008) investigated the effect of end-condition in depth, showing that the use of the channel floor as an end-condition removes the intermittency in the upper and lower branch transition of VIV. They concluded that this is likely due to boundary layer effects of the water channel.

The three conditions tested were: the circular cylinder placed within $0.1 D$ of the channel floor, the cylinder raised off of the channel floor and outside of the influence of the boundary layer with no end-plate attached, and the same configuration but with an attached end-plate. All of these end-conditions promote parallel vortex shedding from the cylinder. All other experimental apparatus remained the same for the runs, with the air-bearing rig raised to lift the cylinder up off of the channel floor.

The results of these tests are shown in figure 2 and allow comparison of the amplitude response between the three end-conditions and the results of Khalak \& Williamson (1997a) and Assi et al. (2006). Khalak \& Williamson (1997a) used a static end-plate raised off the channel floor to within $0.04 D$ of the oscillating cylinder's end (as discussed in Khalak \& Williamson (1996)), while Assi et al. (2006) placed their cylinder at $0.06 D$ of the channel floor.

The mass ratio of our cylinder placed close to the channel floor is $m^{*}=2.2$, comparable with both Khalak \& Williamson $(1997 a)\left(m^{*}=2.4\right)$ and Assi et al. (2006) $\left(m^{*}=1.9\right)$, while the mass-damping parameters were $m^{*} \zeta=6.49 \times 10^{-3}$, 0.0108 and 0.013 , respectively. The damping was determined using (1.2) for both Khalak \& Williamson (1997a) and the present study, however Assi et al. (2006) employed the 'structural' damping without consideration of added mass. The Reynolds number range was similar for all tests, with the current work undertaken over the range $R e=2500-12500$. The effect of $R e$ on VIV has been discussed previously by Govardhan \& Williamson (2006) and Klamo et al. (2006). For our tests with the cylinder raised, the mass ratio increased to $m^{*}=3$ causing lower maximum amplitudes and shorter synchronization regions (Sarpkaya 2004).

All present measurements were taken with the velocity increasing between tests, and the hysteresis phenomena between the initial and upper branch (revealed in Khalak \& Williamson 1997a) was not investigated in this study.

For both of the raised cases tested, the time histories show the recovery of the intermittency of the upper and lower branches as seen in the discontinuous amplitude branch transition found by Khalak \& Williamson (1997a), although with varying time scales of intermittency. A second result of our tests and that of Khalak \& Williamson (1997a) is that the amplitude of the lower branch correlates not with mass ratio but with whether or not an end-plate is used. The $A_{\max }^{*}$ envelope of the attached end-plate has the same amplitude lower branch, $A_{\max }^{*}=0.6$, as that of Khalak \& Williamson $(1997 a)$. Without end-plates there is a higher amplitude of $A_{\max }^{*}=0.7$. These results agree with those of Morse et al. (2008), and highlight how strongly the end-conditions affect VIV. Assi et al. (2006) found no distinctive lower branch in their results, which retained the monotonic decrease from the upper branch through to desynchronization. This is likely to be due to the difference in their definition of damping ratio. 

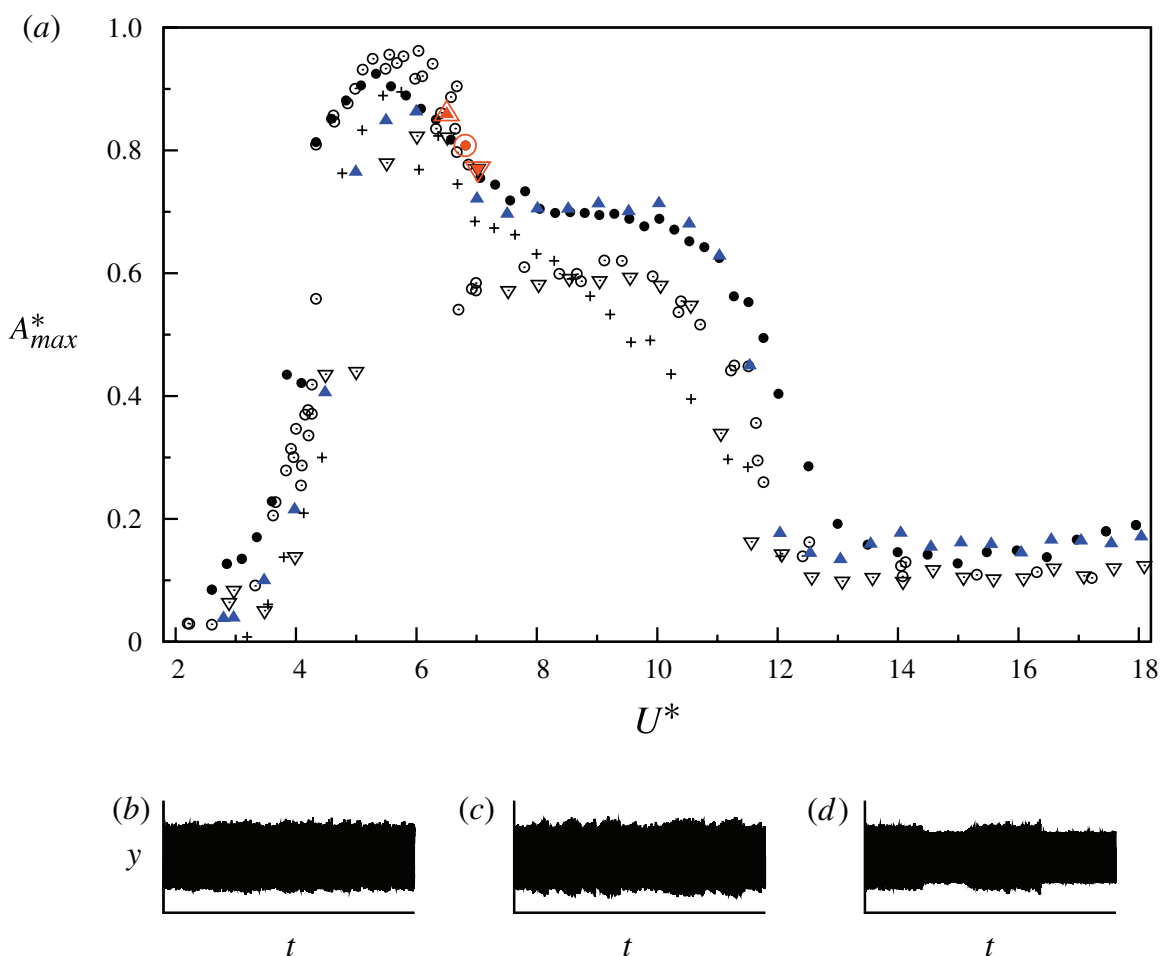

(c)
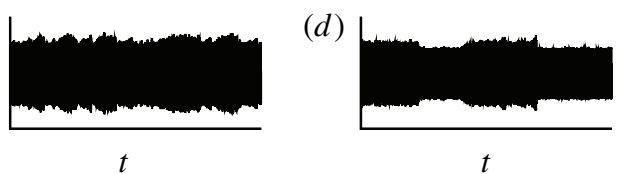

FIgURE 2. (Colour online) (a) Comparison of $A_{\text {max }}^{*}$ against reduced velocity, $U^{*}$, for various end-conditions. Filled circles denote circular cylinder placed near channel floor $\left(m^{*}=2.2\right)$, filled triangles denote circular cylinder $\left(m^{*}=3\right)$ raised off of the channel floor, open triangles denote raised circular cylinder with end-plate attached $\left(m^{*}=3\right)$, open circles denote Khalak \& Williamson $(1997 a)\left(m^{*}=2.4\right)$ and crosses denote Assi et al. (2006) $\left(m^{*}=1.9\right)$. Time histories of cylinders at the end of the upper branch (highlighted symbols in the amplitude response, shown in red in the online version) for $(b)$ channel floor, $(c)$ raised and $(d)$ raised with attached end-plate configurations.

These results provide one explanation for the large variations in reported amplitude responses across different experimental studies. For the remainder of the present study the water channel floor was taken as the end-condition to permit parallel shedding.

\section{Vortex-induced vibration of a square cylinder at $\alpha=45^{\circ}$}

The FIVs of the square cylinder are first investigated for the limiting cases of $\alpha=45^{\circ}$ (referred to as the diamond) and $\alpha=0^{\circ}$ (referred to as the square). The diamond response behaviour and wake vortex shedding modes are compared against the well-known response of the VIV of a circular cylinder. The corners of the diamond shape enforce symmetric separation points located at the vertical centreline of the body resulting in a considerable afterbody. These features are comparable with those of the circular cylinder and produce a similar wake morphology.

\subsection{Amplitude response}

In the previous section the $A_{\max }^{*}$ scalar was used primarily because it provided a basis to validate the results against those of other studies. To improve our comparison of the 
(a)

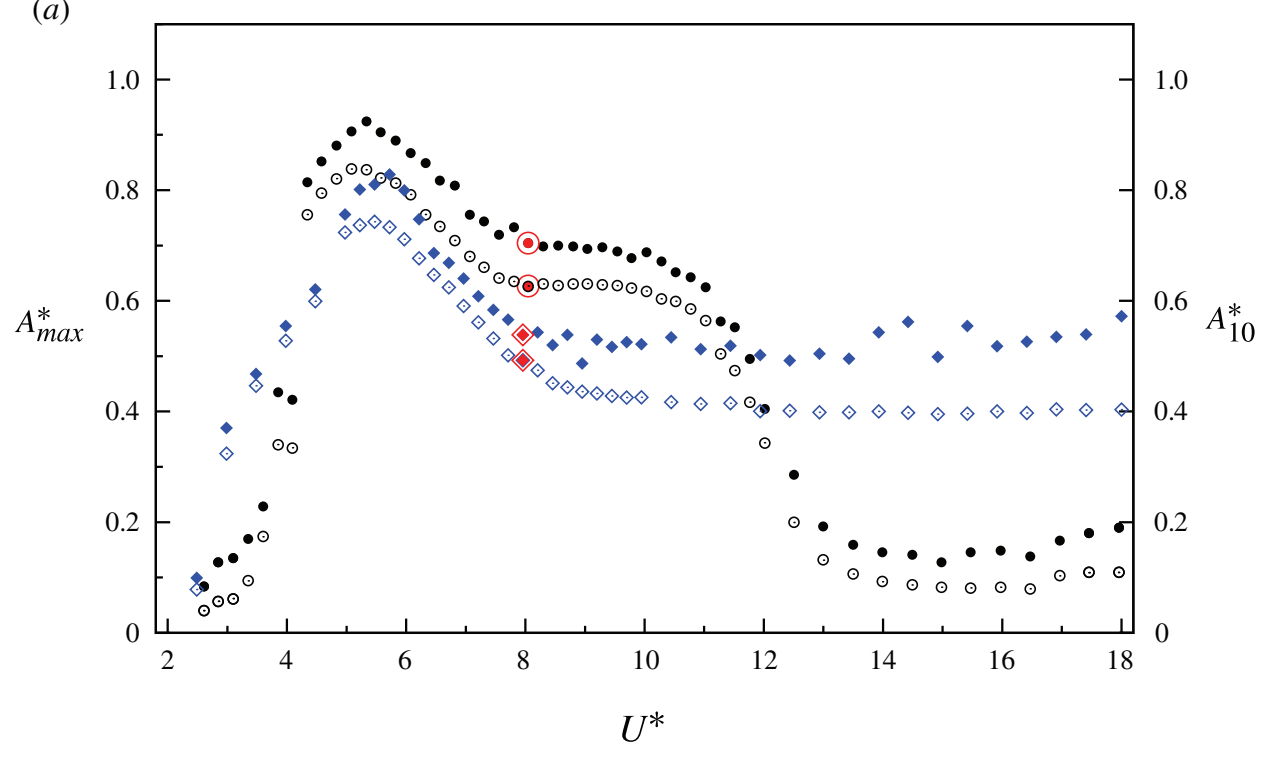

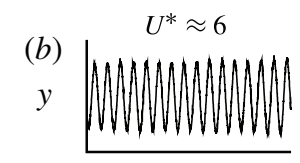

(c)

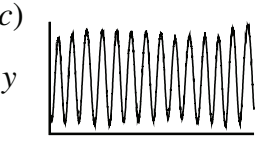

$t$


$t$
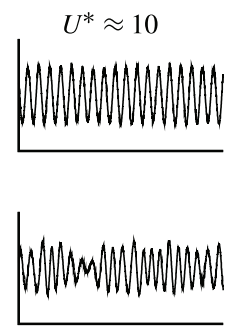

$t$
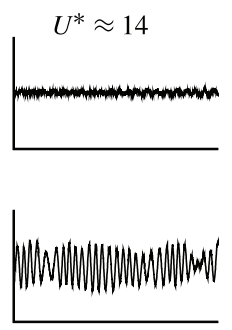

$t$

FIgURE 3. (Colour online) (a) Comparison of reduced amplitude response against reduced velocity, $U^{*}$. Circles represent a circular cylinder $\left(m^{*}=2.2\right)$, diamonds represent a square cylinder at $\alpha=45^{\circ}\left(m^{*}=2.2\right)$, with filled (hollow) symbols denoting $A_{\max }^{*}\left(A_{10}^{*}\right)$ value. Time histories of $(b)$ circular cylinder and $(c)$ diamond cylinder displacement illustrate the loss of periodicity in the oscillations with increasing $U^{*}$.

amplitude response between geometries, a new scalar for the amplitude response, $A_{10}^{*}$, is introduced. It is defined as the mean of the top $10 \%$ of the peaks, as used by Hover, Techet \& Triantafyllou (1998) and Morse et al. (2008). This scalar provides a more appropriate and statistically robust measure for comparison of different, independent studies and across different geometries. The $A_{\max }^{*}$ gives an extremum measure that is the value of a single sample. It can denote an outlier that is not necessarily representative of the average of a larger sample and hence the behaviour of the amplitude response. The two scalars are both valuable for engineering applications, where the $A_{\max }^{*}$ is primarily of importance to determine design conditions for the deflection of structures and supports, while $A_{10}^{*}$ can provide a more meaningful value for assessing the typical amplitudes to consider in determining cyclic loading fatigue.

The $A_{10}^{*}$ amplitude response is shown in figure $3(a)$ along with the $A_{\max }^{*}$ for reference. The freely oscillating diamond exhibits an amplitude response pattern that initially compares well with the circular cylinder undergoing VIV. Starting from a 
very low amplitude of oscillations, $A_{10}^{*}=0.1$ at low velocities, the diamond response increases to a resonant upper branch similar to that of the circular cylinder, without a clear distinction between initial and upper branches. The upper branch begins at $U^{*}=1 / S t$ for both geometries. The value of the $A_{10}^{*}$ peak of the diamond is $A^{*}=0.75$, lower than for the circular cylinder used in the current study. However, it must be remembered that the amplitudes are normalized by the characteristic length, $H$, complicating direct comparisons between the amplitudes of the two geometries. Both bodies follow the same gradual decrease from the upper branch with no intermittent behaviour, due to the influence of the end conditions. At velocities above $U^{*}=8$ the maximum amplitude remains in the range $A_{\max }^{*}=0.5-0.6$ until the end of the velocity range, diverging from the circular cylinder behaviour, which experiences a drop in amplitude to small oscillations when the vibrations and vortex shedding desynchronize.

The lower branch $A_{10}^{*}$ levels out and maintains a constant value of $A_{10}^{*}=0.4$ over the remaining velocity range. There is a slight amplitude drop noticeable at $U^{*}=12$, close to the $U^{*}$ of the desynchronization of the circular cylinder but this appears to be coincidental. The time histories in figure $3(b-c)$ indicate that the diamond cylinder's motion gradually loses its periodicity as the reduced velocity moves away from the resonance region. There is no clear boundary of synchronization, as seen for a circular cylinder.

The diamond orientation clearly undergoes a VIV response. The current results suggest that, compared with the classical three-branch response of the circular cylinder, the diamond's amplitude increases to the upper branch more gradually with no independent initial branch for the same $m^{*}$. Furthermore, the lower branch does not exhibit the highly periodic oscillations of the circular cylinder up to a velocity at which it desynchronizes, with a consequent amplitude drop. Instead, the oscillations show a gradual loss of periodicity while the body maintains the lower branch amplitude and extends it to the highest velocities tested in the current study.

\subsection{Frequency and phase response}

The diamond and circular cylinder's reduced frequency and phase response to increasing reduced velocity are shown in figure 4.

Comparing the response of the circular cylinder from the present study (filled circles) with that of Khalak \& Williamson (1997a) (open circles), we see both exhibit identical classical VIV frequency behaviour for low mass-damping. At low $U^{*}$, the circular cylinder's motion initially locks to a constant velocity-frequency gradient, which by definition is the non-dimensional oscillation frequency of the cylinder, $f^{*} / U^{*}$. For VIV this is equal to the shedding frequency of the cylinder, St $\approx 0.207$ for circular cylinders over the Reynolds number range of these experiments. A secondary component in the oscillation frequency also exists close to that of the body's natural frequency (seen in the inset of figure 4). At the start of the VIV upper branch the two oscillation modes merge into a single mode with a frequency peak just under $f^{*}=1$ at $U^{*}=4.25$. This is the $U^{*}$ at which the vortex phase also jumps (Govardhan \& Williamson 2000). The reduced frequency remains close to unity as the amplitude response reaches its maximum value in the upper branch, just after $U^{*}=5$. When the oscillation amplitude starts to decrease from the upper branch peak, it is matched by a linear increase in the reduced frequency of constant gradient, $S t$, that is slightly below that of the stationary cylinder's shedding frequency. For the circular cylinder in the current study, the frequency diverges from the constant St line and locks instead to a constant frequency of oscillations at $U^{*}=8$, coinciding with the start of the constant amplitude response of the lower branch. The reduced frequency remains 

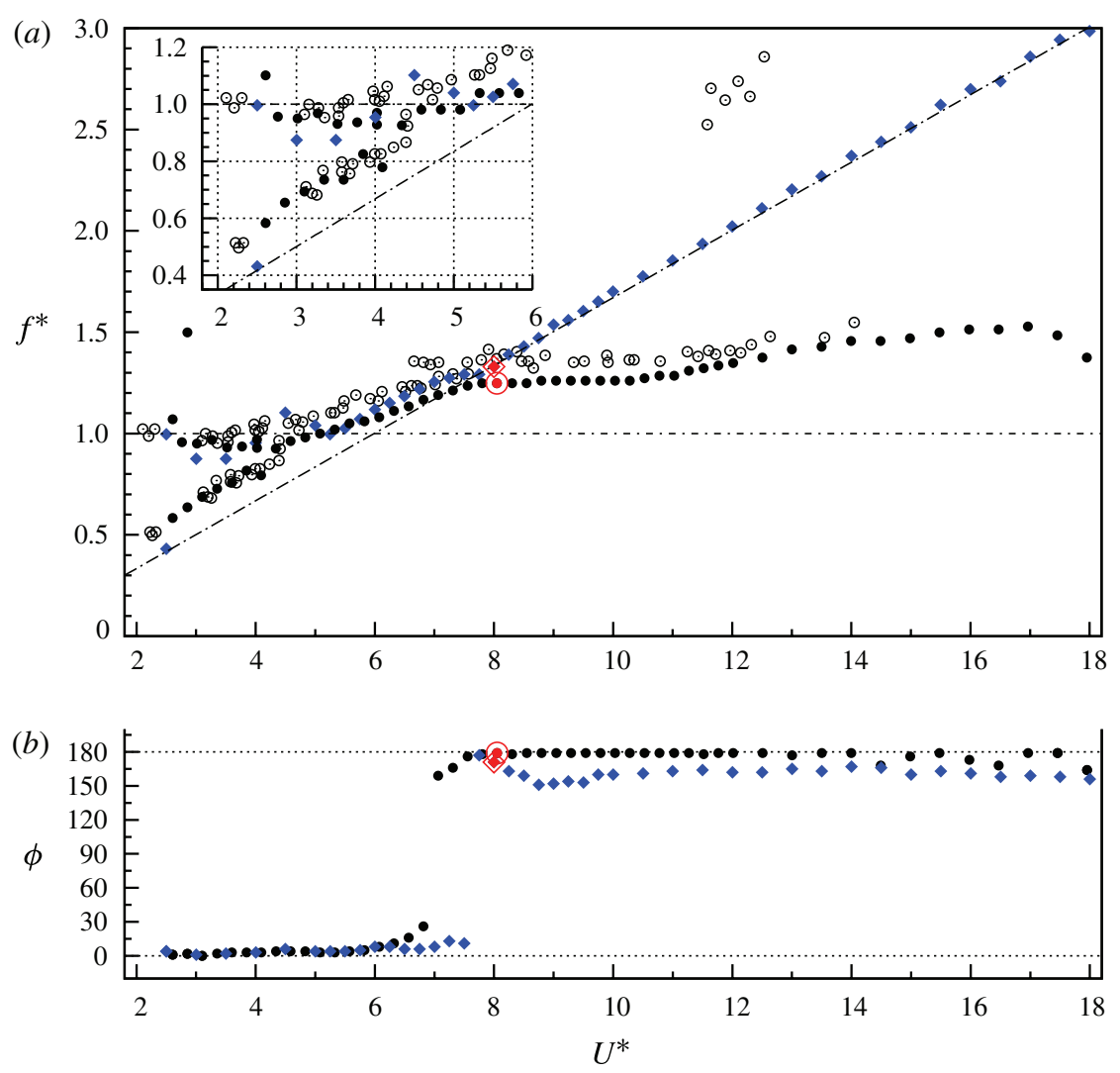

FIGURE 4. (Colour online) (a) Comparison of reduced frequency response, $f^{*}$, against reduced velocity, $U^{*}$. Open circles represent the circular cylinder of Khalak \& Williamson (1997a) $\left(m^{*}=2.4\right)$, filled circles represent the circular cylinder of the present study $\left(m^{*}=2.2\right)$ and filled diamonds represent the square cylinder of the present study at $\alpha=45^{\circ}$ $\left(m^{*}=2.2\right)$. The guideline, $S t=0.167$, is the shedding frequency of the stationary diamond shape. (b) Response of the total phase angle, $\phi_{t}$, between the dominant frequencies of the transverse force and the body displacement.

locked to $f^{*}=1.3$ throughout the lower branch of oscillations. This compares well with the work of Khalak \& Williamson (1997a), who found that the frequency locked to a value of $f^{*}=1.4$. The difference in lock-in frequency is likely to be a result of the different end-conditions.

The vibrating circular cylinder of the present study loses both synchronization and its natural frequency lock-in at a reduced velocity of $U^{*}=10$, coinciding with the end of the lower branch and a consequential drop to smaller oscillations $\left(A_{\max }^{*}<0.2 D\right)$. For velocities above this value the frequency increases nonlinearly with velocity, and the response has no clear relationship to either the shedding or natural frequency of the body. For the two circular sections the timing and length of the frequency lock-in compare well, as expected for similar mass ratios (Govardhan \& Williamson 2004).

The corresponding total phase, $\phi_{t}$, between the dominant transverse force and the displacement remains in-phase, just above $\phi_{t}=0^{\circ}$, throughout the initial and upper branch before jumping sharply out of phase to $\phi_{t}=180^{\circ}$ after $U^{*}=7$. This is linked to the upper-to-lower-branch transition and occurs at the point where the frequency 
passes through the natural frequency of the body in air, $f=f_{N a}$ (Govardhan \& Williamson 2000). In the present study the circular cylinder's oscillation frequency is equal to the measured natural frequency in air, $f_{N a} / f_{N}=f / f_{N}=1.17$, over the same reduced velocity range as this phase jump (see figure 3).

The frequency response of the diamond cylinder does not lock on to a constant $f^{*}$ in the lower branch as evidenced by the time histories in figure $3(c)$ and in the frequency response. Between $U^{*}=2.5-8$ the frequency of the diamond section responds in a similar way to that of the circular cylinder. At the lowest velocity, $U^{*}=2.5$, the diamond experiences two frequencies in the oscillations: one at the natural frequency and a second lying on the shedding frequency slope of the diamond at this Reynolds number range, $f^{*} / U^{*}=S t=0.167$. For all other velocities the oscillations are dominated by a single frequency. The response up to $U^{*}=5$ is clearly influenced by the natural frequency, $f_{N}$, with values fluctuating about $f^{*}=0.8-1.2$ and coinciding with the transition to the upper branch on the amplitude plot. The next region is an St line of slightly decreasing gradient, shared with the circular cylinder response, and matched by the amplitude decrease from the peak to the lower branch. The similarity in frequency response between the two geometries end here. While the circular cylinder locks to a constant frequency in the lower branch, the diamond oscillations are dominated by the vortex shedding Kármán frequency enforced by the fixed separation points and locked to the slope, $S t=0.167$.

The phase relationship for the diamond shows the same $\phi_{t}=180^{\circ}$ jump as the circular cylinder. This occurs at the velocity where the oscillation frequency locks to the Strouhal curve and assumes the characteristics of the lower-amplitude branch over a very narrow reduced velocity range of $U^{*}=7.5-7.75$, after which it dips to $\phi_{t}=150^{\circ}$ before fluctuating around $\phi_{t}=170^{\circ}$. The frequency and phase responses of the diamond are clearly driven by VIV. However, the geometry weakens the coupling of the body's oscillation to its natural frequency and the oscillations remain fixed to the timing of the vortex shedding throughout the lower branch. The most striking result is that, compared with the circular cylinder, the diamond geometry promotes a near-constant amplitude of oscillations driven at the shedding frequency. This occurs for all velocities above the resonant upper branch region.

\subsection{Wake modes}

Recent investigations into oscillating circular cylinders have studied the vortex structure in the wake, resulting in maps and classifications of vortex modes for forced (Carberry et al. 2004; Leontini et al. 2006) and freely vibrating cases (Govardhan \& Williamson 2000). In the low-damping freely vibrating cases, as investigated here, the vortex formation for a circular cylinder changes from two single vortices shed over a cycle to two vortex pairs, a switch from a $2 \mathrm{~S}$ to a $2 \mathrm{P}$ wake mode using the classification of Williamson \& Roshko (1988). This switch is located at the point of transition from the initial to the upper branch in the amplitude response. The exact nature of the switching between the vortex modes and its relationship to dynamics of the system is currently unresolved.

Vorticity contours of the wake mode of the diamond cylinder are shown alongside those of the circular cylinder in figure 5 for a reduced velocity of $U^{*}=8$. The sequence of phase-averaged sets of vorticity contours (see (i)-(iv) in figure 5a) recovers the known $2 \mathrm{P}$ vortex shedding mode for the circular cylinder, as captured originally by Govardhan \& Williamson (2000), for the low-mass-damping lower branch response. The figure shows one oscillation cycle at four phases, with dashed isolines representing negative, clockwise $(\mathrm{CW})$ vorticity and solid isolines representing 

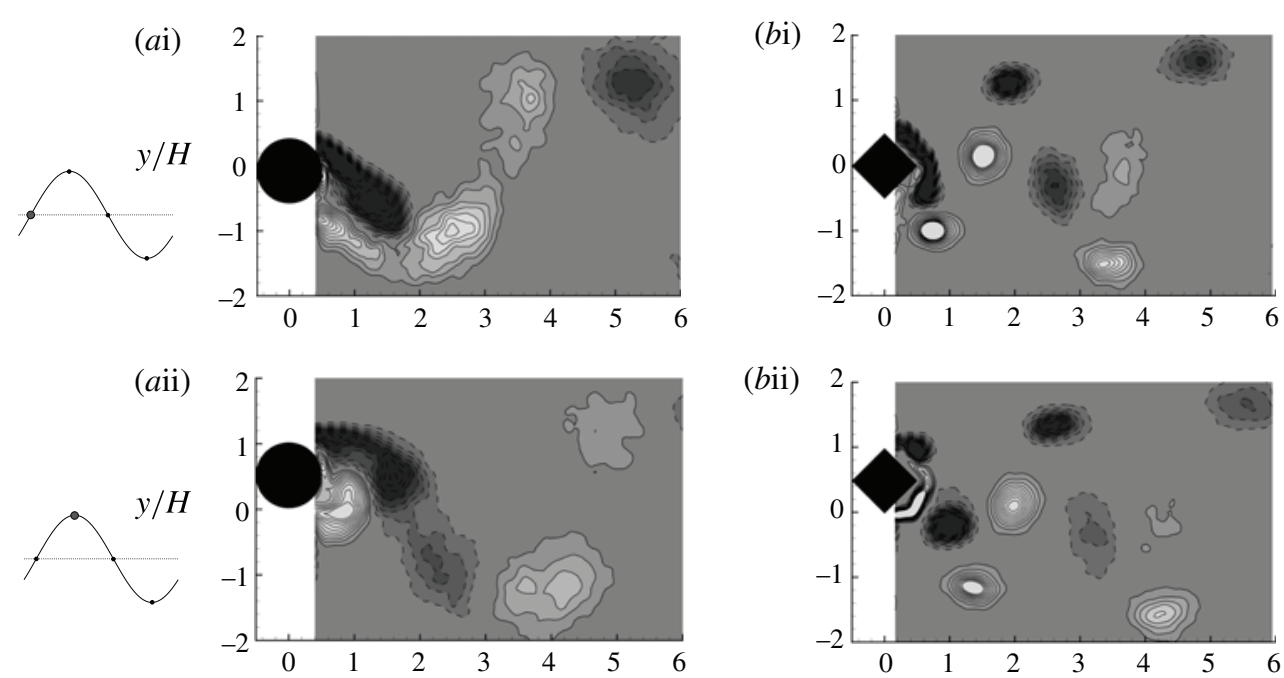

(bii)
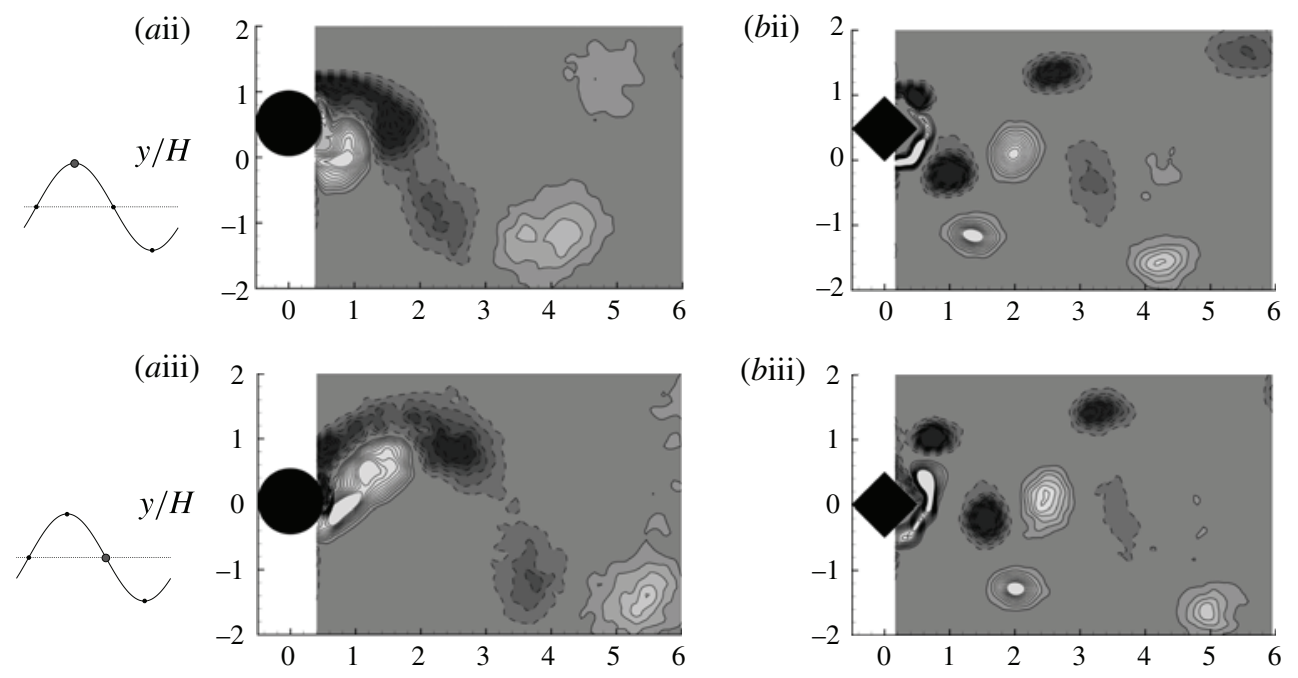

(biii)
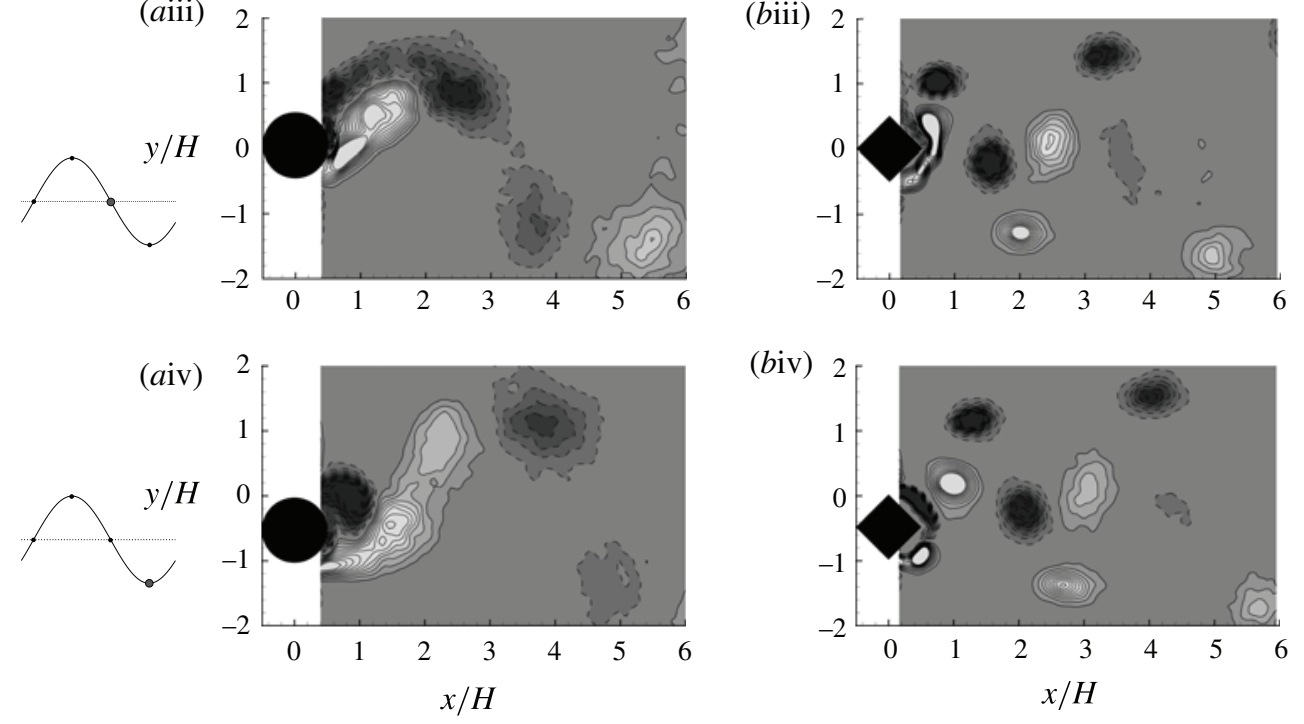

FIGURE 5. Vorticity plots of the lower branch $2 \mathrm{P}$ shedding modes of $(a)$ the circular cylinder and $(b)$ the diamond cylinder at $U^{*}=8$, denoted by the highlighted symbols in the amplitude and frequency responses in figures 3 and 4, respectively (shown in red in the online version). Four phases are shown over one oscillation cycle, from top to bottom, locations shown on the left. The dashed isolines represent clockwise (negative) vorticity, solid isolines are counterclockwise (positive) vorticity.

positive, counterclockwise (CCW) vorticity. At the beginning of the oscillation cycle, (see (i) in figure 5a), the cylinder is moving up and crosses the centreline in the positive direction, shedding a CCW vortex of from the bottom shear layer. CW vorticity attached to the top shear layer is drawn across the centreline of the wake. As the body reaches the top of the oscillation cycle in (ii) the top shear layer sheds a weak vortex that is advected downstream in the wake and pairs with the stronger vortex of opposite sign (shed in (i)) forming a counter-rotating vortex pair. The process 
is mirrored about the centreline in (iii)-(iv) as the negative sign shear layer moves back across the centreline and sheds a strong CW vortex (iii), separating as the positive vorticity moves upwards into the shear layer. This is paired by a weaker CCW vortex (iv) as the body finishes one oscillation cycle. This final pair is observable in the far wake of the first phase of the next cycle (shown in (i)). The secondary weak vortex in these pairs is shed due to the stretching of the shear layer across the centreline as the body changes direction. The stronger vortices are separated from the shear layers in the high strain field where large vorticity of opposite signs interact behind the body.

The diamond cylinder reveals the same $2 \mathrm{P}$ shedding mode (see (i)-(iv) in figure $5 b$ ) as the circular cylinder, albeit with different features. The corners of the diamond fix the location of the shear layer separation and so determine where the vortex roll up begins, while the sharp afterbody creates a high strain field behind the trailing edge. This results in more concentrated vortex cores that separate from the shear layers closer to the body than for the circular cylinder. This earlier spatial evolution of the vortex splitting also leads to a different organization of vortices in the far wake. The same vortex shedding phases are used as above with respect to the circular cylinder In the first phase (i) the negative vorticity around the body crosses the centreline and splits in the high strain field at the trailing edge. It then advects downstream (ii) near the centreline with only a streamwise component of motion. The CCW vortex closest to the body is advected with a transverse velocity component and moves outward from the centreline, expanding with the wake. As for the circular cylinder, this is mirrored along the centreline in (iii)-(iv) with vortices of the opposite sign. The first vortex shed from the shear layers in each phase cycle is weaker than the second vortex. However, in the diamond's wake they do not pair with vortices of the opposite sign, forming instead a four row vortex arrangement over the first $5 H$ of the wake length. In this region the wake is characterized by strong vortices that expand out into the wake and have vorticity of the same sign as the shear layer on that side of the wake. Weaker vortices advect downstream in a path that remains close to the centreline, in a line of alternating sign.

The phase-averaged plots show that the vortices from the diamond cylinder are more compact than those seen in the wake of a circular cylinder. This, at least in part, can be attributed to two factors: the vortices have smaller cores because of how they are generated, and because there is less variation in the position of the vortices across the individual PIV snapshots. Both factors arise from the body's geometry and its fixed separation points. The difference in vortex spacing between the two bodies can also be attributed to differences in the characteristic length, $H$, which appears in both the length scale and the reduced velocity, $U^{*}$. Additional wake visualizations (not shown) revealed that the wake transition from a $2 \mathrm{~S}$ mode to $2 \mathrm{P}$ occurred over the range $U^{*}=3.75-4.25$ for both body geometries.

\section{Square cylinder at $\alpha=0^{\circ}$}

The FIVs discussed above are for one limiting case, the diamond orientation, and revealed a VIV response that compared well with the VIV of a circular cylinder. The second limiting case for the square cross-section is at $\alpha=0^{\circ}$, the square orientation. This configuration shares the same symmetries as the diamond case, but has been shown to be susceptible to galloping at a similar reduced velocity range for lowmass-damping configurations (Bearman et al. 1987). Galloping of square cylinders is 

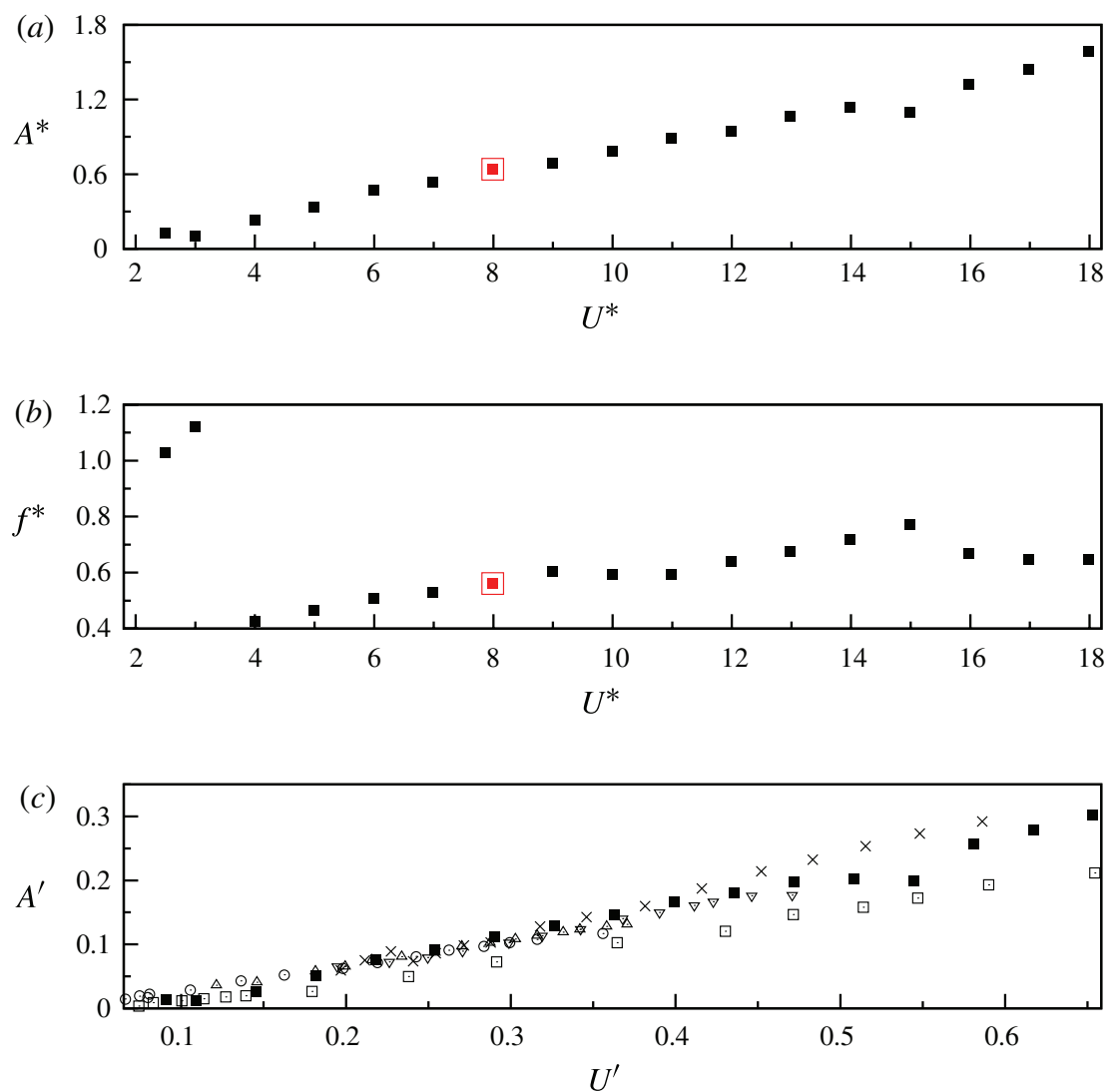

FIGURE 6. (Colour online) (a) Reduced maximum amplitude response, $A_{\max }^{*}$, and $(b)$ reduced frequency response, $f^{*}$, for the square cylinder at $\alpha=0^{\circ}$ against reduced velocity, $U^{*}$. The highlighted symbols (shown in red in the online version) are locations where the wake structures were captured. $(c)$ Validation of the current response (filled squares) renormalized and compared with galloping data from Parkinson (1989). Circles represent $m^{*}=15.1$, triangles represent $m^{*}=9.6$, inverted triangles represent $m^{*}=5.3$ by Bouclin (1977), crosses represent $m^{*}=5.1$ by Santosham (unpublished work used in Parkinson 1989) and open squares represent $m^{*}=8.2$ by Bokaian \& Geoola (1983).

briefly reviewed here using the present experimental facility, primarily to validate and to contrast it with the VIV seen in the diamond case.

\subsection{Amplitude and frequency response}

The classical galloping response of FIVs of the square is shown in figure 6, with the amplitude response recovering the expected linear response predicted by quasi-steady theory of Parkinson \& Smith (1964).

Figure $6(c)$ is a plot of the amplitude response from reported experimental galloping studies. To account for varying mass ratios the amplitude is renormalized as $A^{\prime}=A / 2 m^{*}$, and the renormalized velocity becomes $U^{\prime}=U / 2 m^{*}$, similar to the method used by Parkinson (1989). The results from the present results (filled squares) agree well with the prior work, which covers a higher range of mass ratios, $m^{*}=5-15$. The variation of amplitudes across the studies is attributable to the different experimental conditions (Parkinson 1989) and turbulence intensities of the 

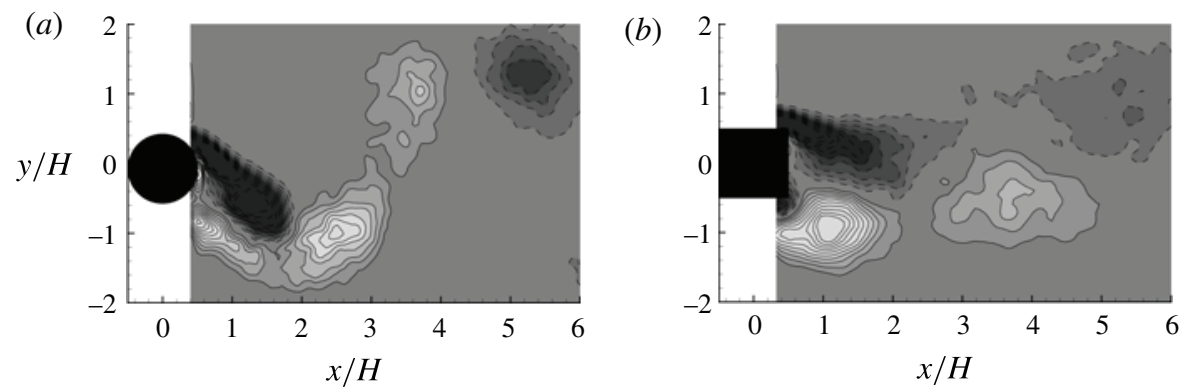

FIGURE 7. Vorticity plots of shedding modes of $(a)$ the circular cylinder in the lower branch $2 \mathrm{P}$ shedding modes and $(b)$ the square cylinder with $2 \mathrm{~S}$ mode at the same reduced velocity $U^{*}=8$.

experiments. A noticeable deviation from the trend occurs around $U^{\prime}=0.55$, where the amplitude deviates from the linear growth: this will be addressed later in this section.

The amplitude response of the square cylinder has a monotonic increase with velocity and, unlike VIV, there is no self-limiting amplitude. Hence, for a reduced velocity above $U^{*}=9$ the $A_{10}^{*}$ amplitudes for galloping are in excess of those achieved in the VIV upper branch, with an amplitude in excess of $A_{10}^{*}=1.5 \mathrm{D}$ at the maximum investigated velocity of $U^{*}=18$. At $U^{*}>14$ there is a slight deviation in the amplitude trend, a 'kink' also observed by Bearman et al. (1987) in low-damping wind tunnel experiments, not predicted by quasisteady theory. Bearman et al. (1987) correlated it with a transverse force component occurring at three times the body oscillation frequency, a result also found in the present study's force measurements. They attributed it to complex vortex formation caused by the high strain imposed by the sharp afterbody corners on the shear layers.

The oscillatory response shows that the initial movement of the square cylinder occurs at frequencies above the natural frequency of the body, $f^{*}>1$. At $U^{*}=4$ the oscillation frequency drops to $f^{*} \approx 0.4$, lower than the vortex shedding frequency and constant with a galloping oscillation. When started from rest, the square cylinder begins to oscillate due to imposed lift on the body from the vortex shedding, prescribing the oscillation frequency for $U^{*}<4$. The classical galloping response of the unstable cross-section becomes the driving flow-induced motion at higher velocities. At low $U^{*}$ the frequency increases until it reaches a value of $f^{*}=0.6$ at $U^{*}=8$, it then remains constant over $U^{*}=9-11$ and then increases again to almost $f^{*}=0.8$ at $U^{*}=15$. This is the peak in the frequency response, after which there is a switch in reduced frequency to $f^{*}=0.6$. This change in response occurs just after the 'kink' in amplitude response.

\subsection{Wake modes}

The vortex wake mode of the square cylinder is compared with the circular cylinder in figure 7 at the same reduced velocity, $U^{*}=8$. The wake structure of a galloping body has vortices shed at both the body's shedding frequency and the body's vibration frequency, with the two vortex system coexisting (Blevins 1990). As a result, the oscillations are not synchronized with the shedding. Separation from the square body occurs at the leading corners, with a shear layer visibly extending to the rear of the body. The square body can be seen to shed large vortices of similar initial size to 
the circular cylinder, which remain attached to the shear layer up to $1.5 \mathrm{D}$ downstream from the afterbody. These vortices diffuse rapidly downstream.

The nature of the wake modes for galloping has received little interest in the literature, unsurprising given that it is not the driving cause of the vibrations. It would be interesting, however, to obtain better insight into the vortex formation in the region of the amplitude 'kink' where the lift force shows a third harmonic. However, the current visualization of position-based phase-averaged vorticity could not capture the cause of the third harmonic in the lift force shedding.

\section{Vibrational response with angle of attack variation}

The previous sections have shown that square cylinders are susceptible to two types of FIVs at symmetric orientations of the body: VIV dominates the oscillations of the diamond and galloping is the vibration mode of the square orientation. This section reports on the effect of angle of incidence on the response of the freely vibrating cylinder, with a particular focus on the transition from VIV to galloping.

Figure 8 provides a map of how square cylinders respond as a function their angle of attack, $\alpha$, and reduced velocity, $U^{*}$. The parameter map highlights three types of FIV modes and regions where they occur. VIV (light region) exhibits frequency responses similar to that of the diamond; galloping (dark region) with frequency and amplitude behaviour associated with that of the square, and a region of VIV response that is referred to as the higher branch (bound by thick line). Angle-velocity combinations where force and position measurements were recorded are denoted by symbols associated with the dominant FIV behaviours (circle, VIV; square, galloping/low frequency; triangle, higher branch). The relative size of the symbols represent the relative normalized CWT power of the oscillation frequency peak associated with the FIV response. Where two symbols are overlaid there exist two modes in the frequency response, with their symbol size representing their relative power contribution to the oscillation response.

\subsection{Amplitude response}

Consistent with the approach used in $\S \S 4$ and 5, the free vibration's dynamics will be characterized using the response of the amplitude to the angle of attack and reduced velocity. The use of amplitude and frequency as the response variables also makes the results particularly relevant for assessing the likely impact on cyclic fatigue. The $A_{10}^{*}$ response to reduced velocity is shown in figure 9 with varying angle of attack. The reduced amplitude-velocity plots are stacked at increments of $\alpha=2.5^{\circ}$, and the $A_{10}^{*}$ amplitude shown is of the dominant frequency of the oscillations at each recorded value. This scalar reveals the influence of the dominant vibrations of the body, not its absolute position. In fact, the angle of attack generates a net mean lift in asymmetric configurations with a resulting non-zero mean in the body oscillation, shown in figure 10 .

The $A_{10}^{*}$ response to velocity and angle of attack in figure $9(a)$ reveals the three distinct responses of the square section: (i) from $\alpha=45^{\circ}$ down to $\alpha=25^{\circ}$ the amplitude response follows that of the diamond orientation, with both upper and lower branches present with the peak $A_{10}^{*}$ ridge of the VIV upper branch highlighted with a grey line (red in the online version); (ii) between $\alpha=0$ and $7.5^{\circ}$, the body's oscillations follow a trend of increasing amplitude with velocity, as seen in galloping of the square section. This response has a distinct boundary at $\alpha=7.5^{\circ}$; (iii) a third region lies between the VIV and galloping dominated regimes. It has a high-amplitude 


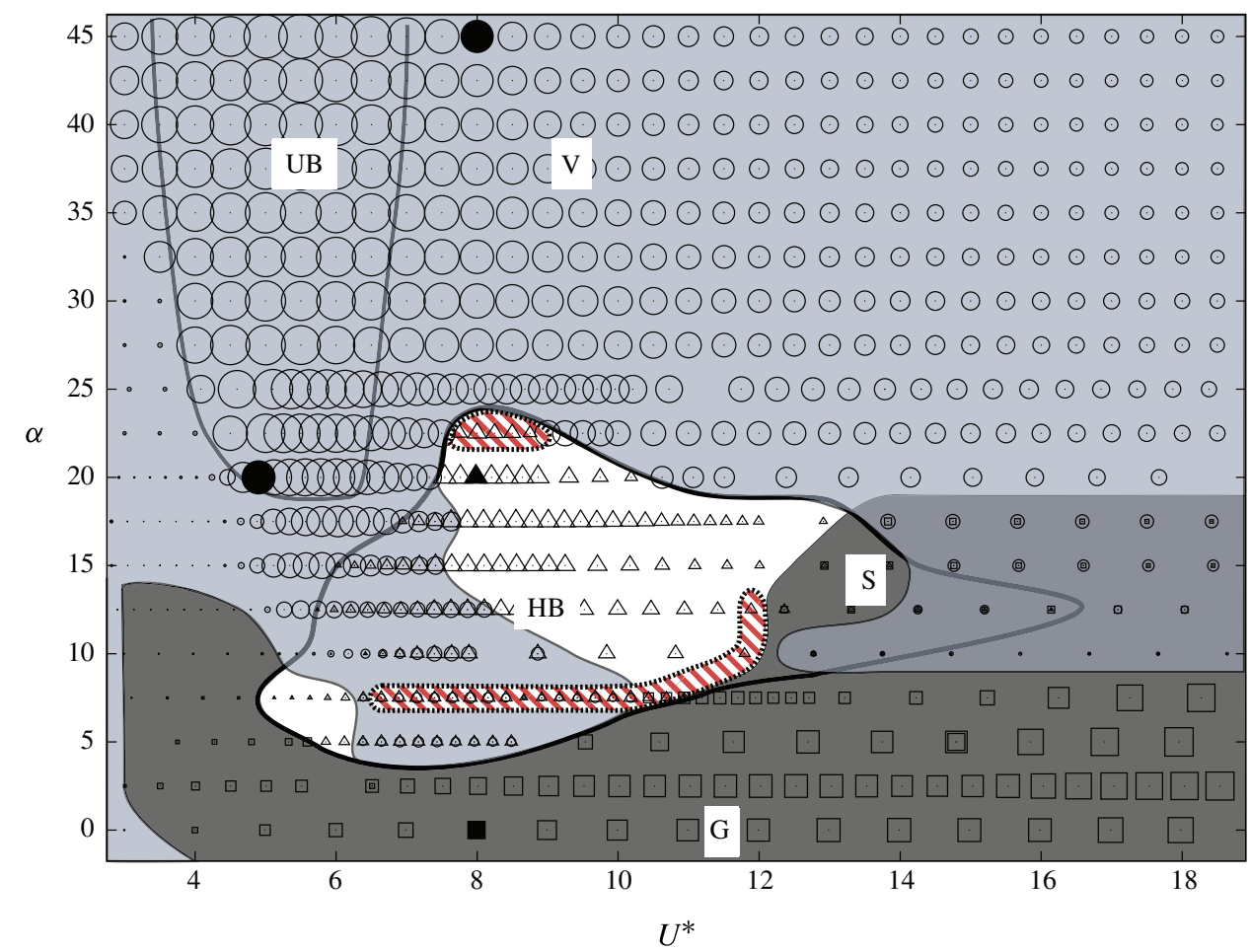

FIGURE 8. (Colour online) Map of FIV response of a square cylinder with variation in the angle of attack, $\alpha$, and reduced velocity, $U^{*}$. Regions are labelled according to response type. The shaded light grey region contains (UB) upper amplitude branch of VIV bounded by solid line and (V) VIV-dominated frequency response; region bound by thick solid line: higher branch (HB) frequency response; dark grey region: galloping response $(\mathrm{G})$ with low-frequency galloping and low-frequency-dominated response with other frequency contributions. (S) signifies part of the parameter space where the HB frequency splits into diverging frequencies. The striped region bounded by dashed borders represents a body oscillation response undergoing intermittency. Parameter space locations where force and position measurements were taken are represented by symbols signifying the dominant frequency of the cylinder motion: shedding frequency (circles); higher branch frequency (triangles); and lower frequencies (squares). The relative size of the symbols denote the relative energy of the dominant frequencies in the system. Filled symbols mark the parameter space where PIV measurements of the near wake were taken.

resonant branch not seen in galloping, and separate to the upper and lower branch documented in VIV studies. This higher branch (HB) reaches a maximum amplitude close to $\alpha=20^{\circ}, U^{*}=8$. The peak ridge of this branch is highlighted with a black line in figure $9(a)$. To highlight the three distinct amplitude responses, discussion will relate the amplitude as a function of reduced velocity for varying angles of attack.

The amplitude response is VIV dominated for angles of attack $\alpha=25-45^{\circ}$ and resembles that of the diamond. The peak value of the resonant upper branch decreases from $A_{10}^{*}=0.76$ to 0.56 as the angle of attack moves away from the diamond configuration. The change in angle also reduces the geometry's shedding frequency that drives the resonant oscillations, and therefore shifts the peak of the resonant upper branch from $U^{*}=5.5$ at $\alpha=45^{\circ}$ to $U^{*}=6.5$ at $\alpha=25^{\circ}$. A consequence of this 
(a)

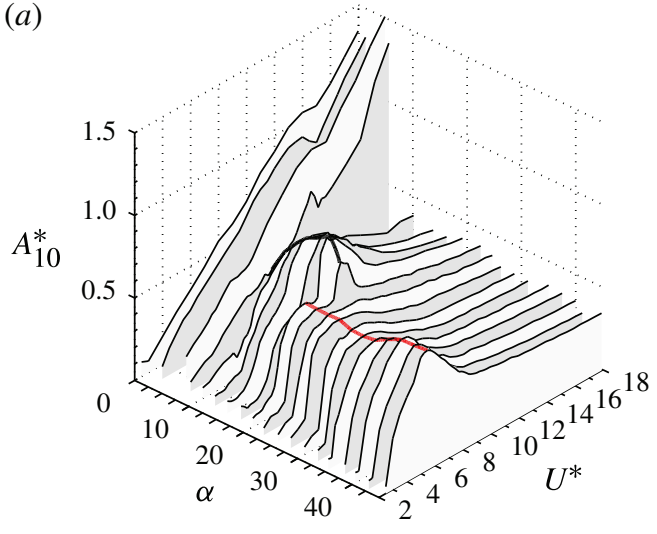

(b)

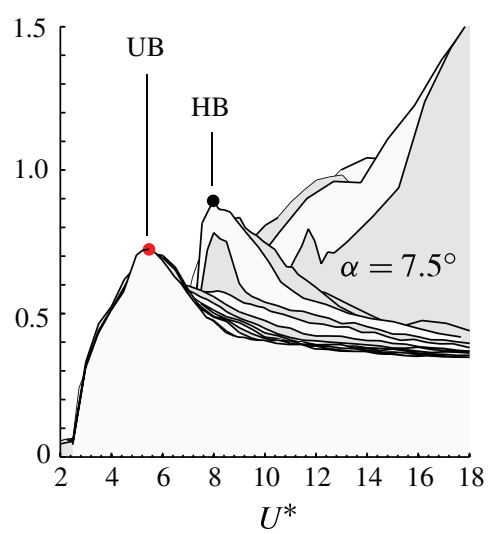

Figure 9. (Colour online) (a) Plots of amplitude response, $A_{10}^{*}$, of the dominant oscillation frequency against reduced velocity, $U^{*}$, and angle of attack, $\alpha$. (b) Amplitude response as a function of reduced velocity with all angles superimposed, with the diamond, $\alpha=45^{\circ}$, at the front. The response curves are in decreasing increments of $2.5^{\circ}$. The sharp transition from a galloping to a combined response is seen in the plots between $7.5-10^{\circ}$. The peak of the VIV upper branch (UB) occurs at $\alpha=45^{\circ}, U^{*}=5.75$, and the new amplitude branch (HB) peak occurs at $\alpha=20^{\circ}, U^{*}=8$.

(a)

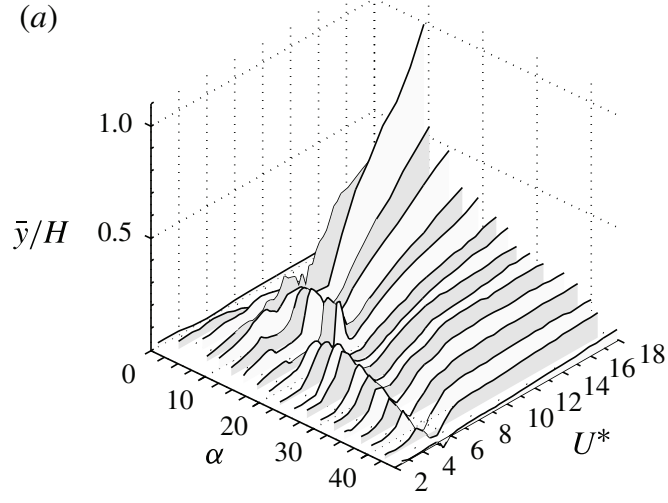

(b)

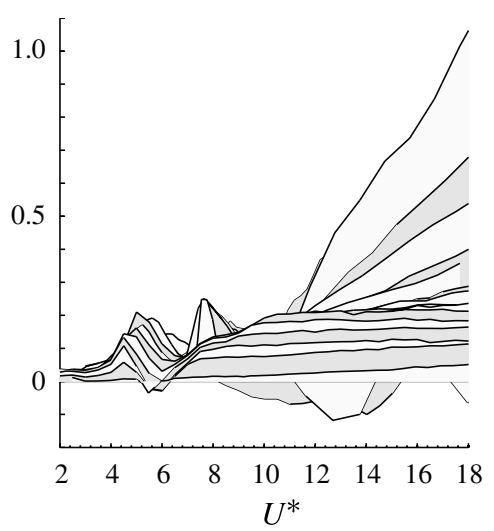

FIGURE 10. (a) Mean amplitude response, $\bar{y} / H$, against reduced velocity, $U^{*}$, for angles of attack, $\alpha=0-45^{\circ}$ at $2.5^{\circ}$ increments, and $(b)$ superimposed plots of the same response. Angles of attack of 2.5 and $5^{\circ}$ show a negative mean amplitude response centred around $U^{*}=13$, while the maximum deviation of the mean from the zero position is seen at $\alpha=10^{\circ}$. The influence of the upper and higher branch on the mean amplitude is visible in the positive peaks centred around $U^{*}=5$ and $U^{*}=8$.

is the onset of the upper branch occurring at increasingly higher velocities, thereby revealing an initial branch in the amplitude response. This branch is similar to that seen in circular cylinder results. The upper-to-lower-branch transition approaches a linear gradient until there is no clear distinction between the two amplitude branches. 
A new amplitude branch is observed in figure 9 over the range of angles $\alpha=10-22.5^{\circ}$. Its amplitude is considerably higher than that seen in the upper branch associated with VIV. The peak amplitude of $A_{10}^{*}=0.9$ is at $U^{*}=8$ and at an angle of $\alpha=20^{\circ}$ and, unlike the upper branch of VIV, the extent of the velocity range of the HB is strongly dependent on the angle of attack. The upper branch and higher branch are seen to co-exist at $\alpha=20-22.5^{\circ}$, and the amplitude increases sharply over a narrow velocity range at the onset of the higher branch, signalling a rapid switch in oscillation mode. This is confirmed in $\S 6.2$ by wavelet analysis. For velocities above those where the HB and UB occur, the $A_{10}^{*}$ oscillation amplitudes decrease with velocity and are consistent in magnitude for the two branches.

Reducing the angle of attack below $\alpha=10^{\circ}$ causes the oscillation amplitude response to change dramatically, as the square orientation is approached and the galloping mode dominates the response. At $\alpha=7.5^{\circ}$ the oscillations no longer follows the lower branch trend of VIV but increase in amplitude with increased velocity: neither the UB nor the HB resonant characteristics are present in the amplitude response. As the angle of attack approaches the symmetric square orientation, the amplitude response smooths out to the response seen in classical galloping response.

The distinct boundary seen in the $A_{10}^{*}$ response separating galloping and the new higher branch regimes only reveals part of the transition. The change in oscillation amplitudes occurs simultaneously with the body's average position moving away from the mean position at rest due to the net mean lift acting on the body (shown in figure 10). The largest offset in the mean occurs at $\alpha=10^{\circ}$, corresponding to the oscillations switching from large-amplitude and low-frequency galloping oscillations to higher-frequency oscillations with smaller amplitudes.

An observation of the mean body position in figure 10 highlights two important features of the mean amplitude behaviour. First, the mean position deviates from the zero position at parameter values matching the VIV upper branch and the higher branch resonant peaks. In these resonant regions the oscillations are dominated by a single frequency with varying amplitude, as seen in the upper branch of low-massdamping circular cylinders. Second, outside of the peak resonant regions, the deviation of the mean from the zero position increases as the angle of attack moves away from the diamond case, due to the square's pressure distribution generating an increasing net lift. The growth is gradual in the VIV-dominated region of $\alpha=22.5-45^{\circ}$, and then becomes rapid in the HB region reaching the largest deviation at $\alpha=10^{\circ}$. At angles of attack below this the mean position recovers as the body approaches the symmetric square cylinder orientation. Unexpected results observed in figure $10(b)$ are the negative values in the mean position of the body at low angles of attack $\left(\alpha<10^{\circ}\right)$, with a deviation from the zero position of up to $\bar{y} / H=-0.1$ for selected reduced velocities in the range $8<U^{*}<15$.

The amplitude response with varying angle of attack clearly reveals the various FIV modes of the square. The oscillation amplitude and mean position show a clear transition in the behaviour from $\alpha=7.5$ to $10^{\circ}$, due in part to the location of the separation points imposed by the corners of the square. As the body is rotated $\mathrm{CW}$ in figure 1, the separation point attached to the top leading corner of the square moves with the geometry's corner towards the centreline. As the angle increases, the flow reattaches to the bottom surface of the square and the bottom separation point shifts from the leading bottom corner to the trailing corner, which in turn approaches the centreline as the body rotates further. The jump in location of the bottom separation point is the probable cause of the transition between the galloping and higher branch behaviour. 

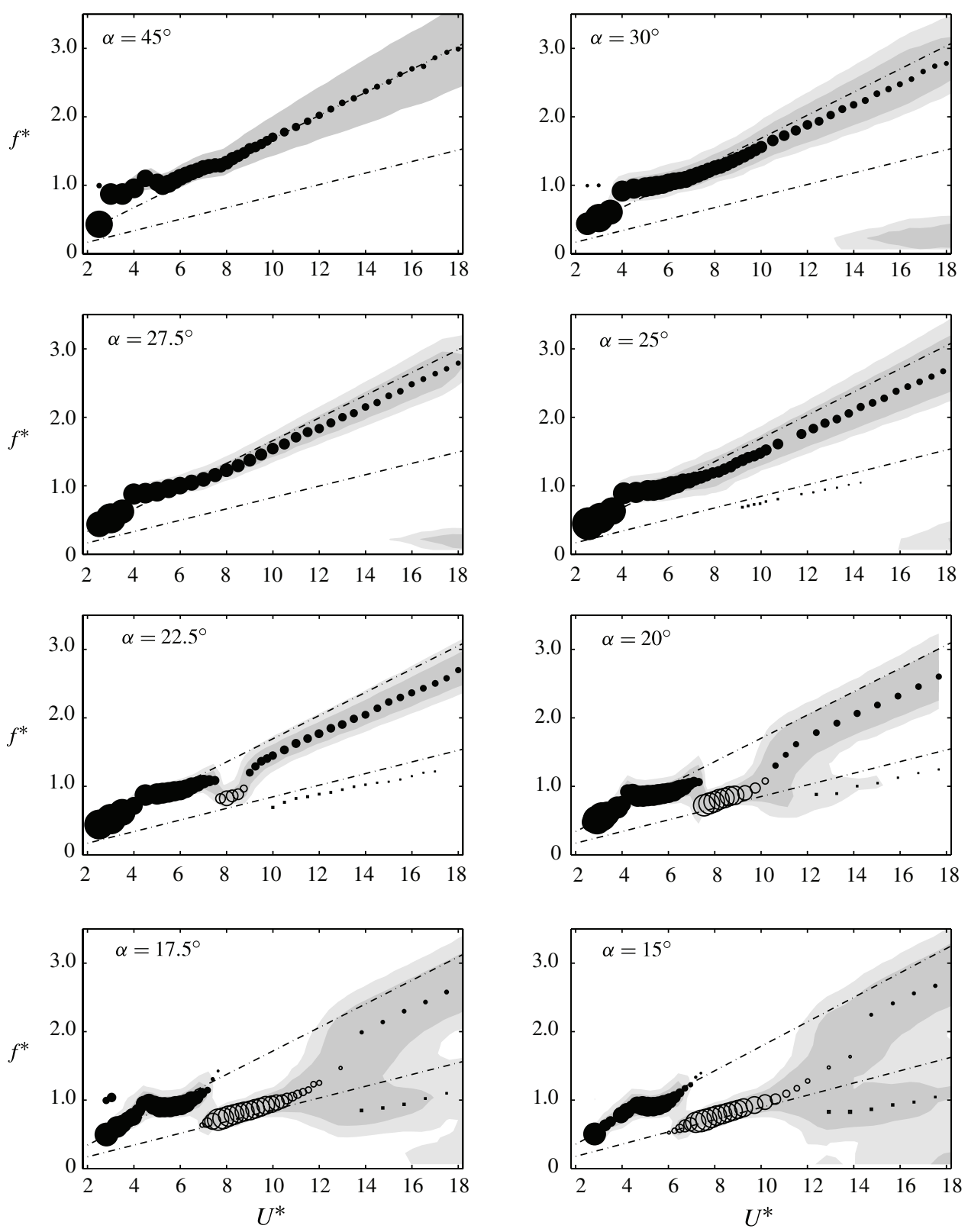

FIGURE 11. For the caption, see the next page.

\subsection{Frequency response}

The frequency responses at different angles of attack are shown in figure 11. The symbols on the plots represent frequency power spectrum peaks and the contours represent the relative normalized energy of the frequency spectra. Symbols are associated with the different frequency response regimes classified as follows: filled circles represent frequencies associated with VIV behaviour, including the body's shedding frequency and those close to the body's natural frequency. The open circles represent frequencies in the higher-amplitude branch. The open squares are for lower 

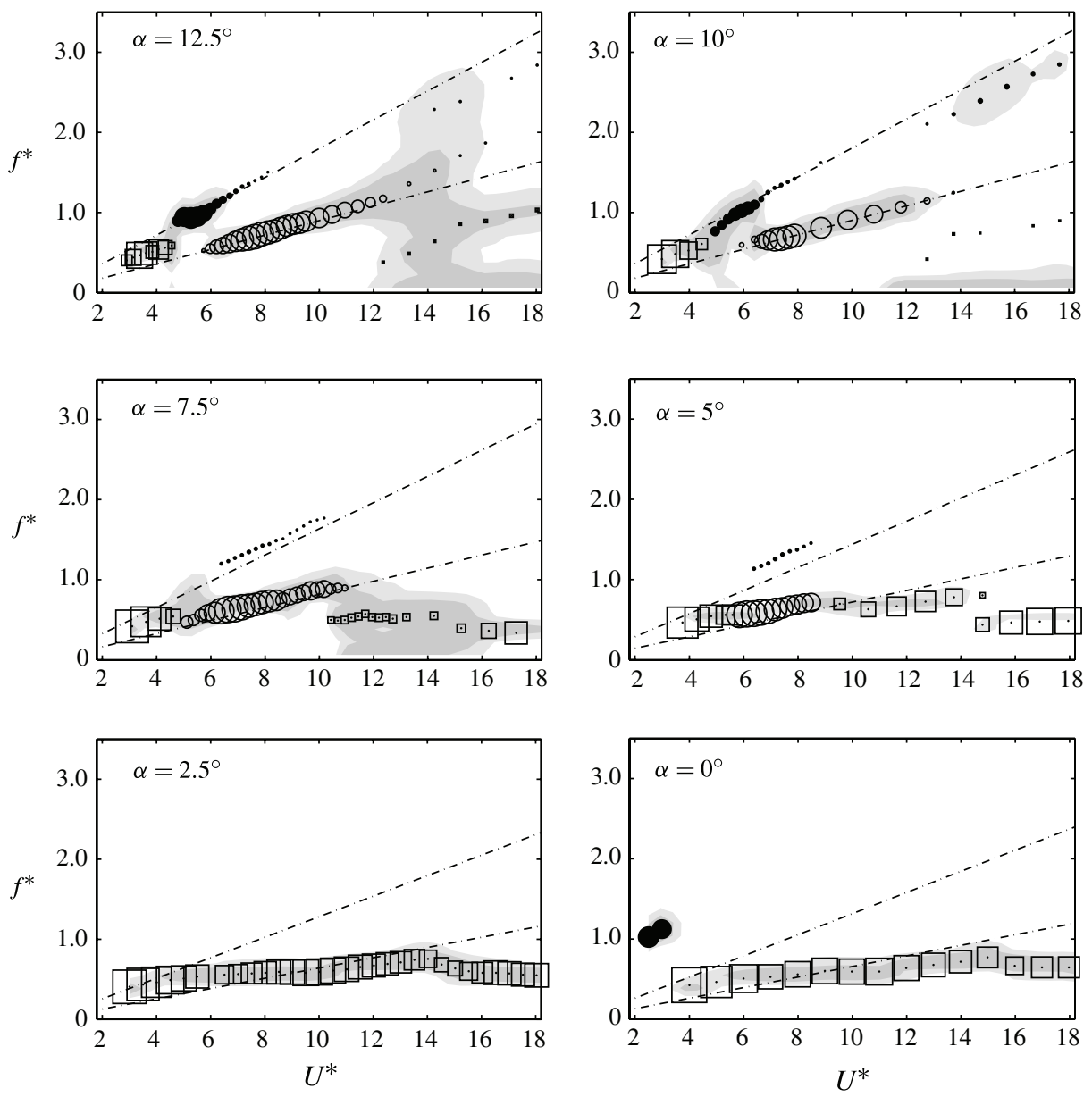

Figure 11. (Cntd) Cylinder oscillation frequency response, $f^{*}$, to increasing velocity, $U^{*}$, at various angles of attack. Shaded regions are contours of relative normalized intensity in wavelet energy. Filled circles represent shedding frequencies, open circles represent frequencies associated with higher branch and open squares represent lower frequencies including those associated with galloping. The size of the symbols relate the power magnitude of the peaks. Dashed lines represent the shedding frequency of the stationary body at the specified $\alpha$ and its first subharmonic.

frequencies associated with galloping oscillations. Symbol size indicates the relative energy contained by the dominant frequency of oscillation at each reduced velocity, a useful visualization aid of the rate of transfer of spectral energy between competing frequency peaks as a function of reduced velocity. The normalized frequency contours superimposed on the plots also reveal this energy transfer as well as indicating the periodicity of the signal. In the case of $\alpha=45^{\circ}$ in figure 11, the contours expand over an increasingly larger frequency range as $U^{*}$ increases, simultaneously the filled circles shrink in size. This can be interpreted as the energy in the system remaining centred around the dominant frequency (the shedding frequency here) but distributed over an increasingly wider band, signalling a loss in the periodicity of the oscillations with increasing reduced velocity. Such insight into the frequency response of the diamond is 
not apparent when inspecting solely the peak frequency values, as shown in figure 4 . The two guidelines in the figures are the stationary shedding frequency of the specified angle and its first subharmonic.

Inspecting the frequency response in the VIV-dominated regime over the domain $\alpha=25-45^{\circ}$ confirms that the body is following the same trend as the diamond cylinder. At each angle of attack within this region, the frequency response to increased velocity follows a line of constant gradient outside of the resonant region of the upper branch. At high reduced velocities the contours reveal low broadband oscillation frequencies, as the VIV lower branch loses periodicity. This low-frequency component is a result of mixed mode behaviour due to the body's susceptibility to galloping.

For velocities in the resonant region of the VIV upper branch, the response also changes with angle of attack. For the diamond orientation, $\alpha=45^{\circ}$, the reduced frequency remains close to unity at low speeds under $U^{*}=5$, however, as the angle is reduced the coupling of the oscillations to the natural frequency weakens and the motion approaches the shedding frequency slope. This is in contrast to the circular cylinder, which experiences both oscillation frequencies within this reduced velocity range (seen in figure 4). Inspecting the other end of the VIV region, at $\alpha=25^{\circ}$, the synchronization with the natural frequency is limited to a narrow reduced velocity region at the onset of the upper branch $\left(U^{*}=4\right)$, after which the frequency monotonically increases at a slope close to the shedding frequency of the body, St. A subharmonic frequency with relatively little energy content can be observed in the range of $U^{*}=9-15$. It will be shown later that this is a result of the mode competition in the fringe region near the higher branch.

At an angle of attack of $\alpha=22.5^{\circ}$ the cylinder motion maintains the shedding frequency except in the region $U^{*}=7.75-9$, where it is dominated by a lower frequency (open circles). This, unsurprisingly, coincides with the higher branch amplitude response and is the associated characteristic frequency trend observed over the range of angles, $\alpha=5-22.5^{\circ}$. It is characterized by an oscillation frequency below that of the VIV response, close to the subharmonic on the Strouhal frequency. The dominant frequency switch from VIV to the higher branch/low-frequency resonance is also angle of attack dependent. At the largest angle of attack at which the higher branch occurs, $\alpha=22.5^{\circ}$, the switch is rapid occurring over a narrow velocity range. In contrast, at $\alpha=12.5^{\circ}$ the spectral energy transfer from the VIV to higher branch is more gradual, occurring over a reduced velocity range, $5.8 \leqslant U^{*} \leqslant 8$. The contours show that the majority of the energy is transferred to the lower frequency by $U^{*}=6.5$. The relative size of the symbols shows that the VIV-associated oscillation frequency is gradually damped out as it is absorbed into a harmonic of the dominant lower frequency. For smaller angles of attack, below $\alpha=10^{\circ}$, the VIV response is no longer present, replaced by a harmonic component of the higher branch seen in $\alpha=5,7.5^{\circ}$. This harmonic suggests that the vortex shedding is influenced by the oscillation mode, and the shedding still influences the body motion but is clearly no longer the dominant cause of vibration.

For angles of attack $\alpha=15-20^{\circ}$, the frequency response in figure 11 reveals the synchronization of multiple modes, resulting in resonance of the higher branch. The frequency associated with this branch undergoes a split into two frequency peaks at higher reduced velocities with diverging slopes of constant St. This trend is clearest at the midpoint of the higher branch region, $\alpha=15-17.5^{\circ}$, as the reduced velocity is increased above $U^{*}>10$. With increasing velocity the power spectrum broadens rapidly and two energy peaks are found; one that approaches the shedding 
frequency of the stationary cylinder, with a second slope at lower frequencies. This switch is preceded by the shift in the frequency response of the lift force on the cylinder to the shedding frequency (not shown). For the higher angles of attack, $\alpha=20-22.5^{\circ}$, the shedding frequency component dominates at higher velocities, but the energy shifts to the lower-frequency slope as the angle of incidence is decreased. No harmonic relationship exists between the two curves, leading to the suggestion that two independent physical mechanisms are at play. It is hypothesized that two FIV phenomena are synchronized in the higher branch resonant region, and the splitting of the frequency is signalling their desynchronization.

At lower angles of attack $\left(\alpha<15^{\circ}\right)$ the response contains multiple low-frequency bands, indicative of complicated fluid-structure interactions. The frequency responses for velocities above the higher branch contain no trace of the shedding frequency, confirming the conclusions drawn from the amplitude response in § 4.1: that the FIV is not driven by VIV at orientations that diverge by only small angles from the square cylinder orientation. A mixed mode of vibration that is a nonlinear combination of galloping and VIV is likely however.

For values below $\alpha=10^{\circ}$ the response contains only low-frequency oscillations as observed in galloping. The kink seen in the galloping amplitude response in figure 9 (also observed by Bearman et al. (1987)) for the angles of $\alpha=0-10^{\circ}$ occurs along with a change in oscillation frequency response. For the square symmetric orientation and the small angle of attack $\alpha=2.5^{\circ}$, there are two low-frequency branches: the first occurs up to $U^{*}=15$ for the square and up to $U^{*}=14$ for $\alpha=2.5^{\circ}$, characterized by a gradual increase in $f^{*}$ with $U^{*}$; the second behaviour is a decrease in frequency approaching $f^{*}=0.5$. These two branches form a continuous trend at $\alpha=0-2.5^{\circ}$, but become discontinuous at $\alpha=5^{\circ}$ (located at $U^{*}=15$ ), leading to a sudden jump in the frequency of oscillations.

\subsection{Wake modes}

Figure 12 shows four phases of the shedding modes for $(a)$ the VIV upper branch and $(b)$ the combined FIV higher branch at $\alpha=20^{\circ}$ over one oscillation period. Of immediate interest from the perspective of circular cylinder studies is the vortex formation for the VIV upper branch. At $U^{*}=5$, the square cylinder is in the centre of the resonant region in the upper branch of VIV, which we have shown in $\S 4.3$ produces a $2 \mathrm{P}$ mode for in the diamond orientation.

At the beginning of the oscillation period (see (i) in figure 12a), the square cylinder is moving upwards across the centreline of the oscillation cycle, and a $\mathrm{CW}$ vortex forms on the topside of the cylinder, with a CCW vortex already shed is behind the cylinder. Near the top of the body motion (ii), CCW vorticity from the bottom wraps around the trailing edge of the body, and the top vorticity sheet splits in the strain region where the vortices meet near the top-right corner of the cylinder, causing the shedding of a CW vortex. The positive vorticity around the body increases (iii) as the body moves back down through the centreline, forcing the top shear layer to shift further from the body. At the bottom of the motion (iv), the top vortex sheet sheds another CW vortex, and vorticity of the same sign develops on the rear of the body, leading to the $\mathrm{CCW}$ vortex that is shed in the first phase of the cycle from bottom corner of the cylinder. Thus, a $\mathrm{P}+\mathrm{S}$ shedding mode is observed with two vortices shed from the top side of the cylinder in each cycle. The first vortex shed from the top side in this phase description of the body motion is significantly weaker than the other two and diffuses rapidly in the downstream wake. In the third phase (iii), the $\mathrm{CCW}$ vorticity on the topside of the square can be assumed to be separated near the 

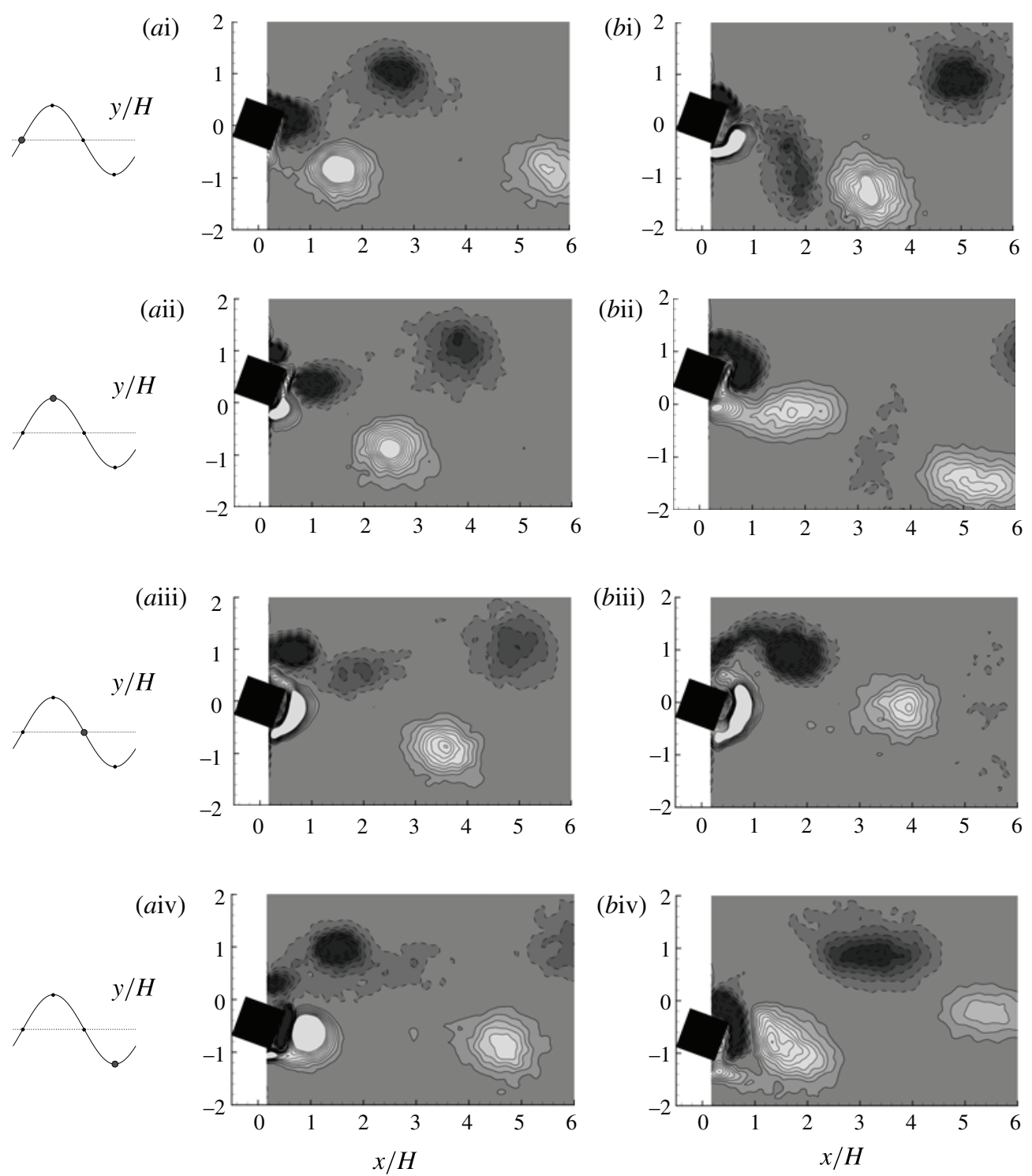

FIGURE 12 . Vorticity plots of the shedding modes of the cylinder oriented $\alpha=20^{\circ}$ in $(a)$ the VIV upper branch at $U^{*}=5$ with $\mathrm{P}+\mathrm{S}$ mode and $(b)$ the higher branch at $U^{*}=8$ with $2 \mathrm{P}$ mode. Four phases are shown over one oscillation cycle for each vortex mode shown from top to bottom, with their phase location in the oscillation cycle shown on the left.

corner of the square and is then cross-annihilated by its interaction with the top shear layer by the fourth phase. These $\mathrm{P}+\mathrm{S}$ near-wake vortex interactions are not revealed in the VIV lift and oscillation frequency response. This is not unusual, as any energy contributed to the body from the vortex splitting of the pair would be minimal. The angle variation is responsible for the location of the square's corners and therefore a determining parameter influencing whether the shear layers encounter the high-strain region located near the corners of the square. The PIV phase images indicate that 
the genesis of the vortex splitting is within these high-strain regions, supporting the observation that the angle of attack defines the wake modes observed. As the square is rotated CCW from $\alpha=45^{\circ}$ (decreasing angle of attack), the rear corner and associated high strain region moves upwards, and less of the bottom shear layer wraps around the body. Eventually, as the body approaches the square orientation each shear layer interacts with a symmetry resulting in the symmetric shedding observed in figure 7.

The higher branch vortex mode, shown in figure $12(b)$ at $U^{*}=8^{\circ}$ and $\alpha=20^{\circ}$ displays a $2 \mathrm{P}$ wake. Comparison of the two resonant regions wake states highlights the difference in timing of the vortex shedding with respect to the body's oscillation. For the higher branch the bottom shear layer in the first phase (see (i) in figure 12b) extends further into the wake than at the lower velocity, where VIV occurs, and results in a splitting that leads to the $2 \mathrm{P}$ wake structure. The first vortices shed from each side are close to the centreline and weaker than the second vortices, as seen for the diamond VIV in figure 5.

\subsection{Intermittency}

It is informative to examine the intermittent behaviour observed in the oscillation response's time series. In the higher branch the square cylinder oscillations undergo an intermittent phenomenon that is not seen in either the VIV or galloping dominated regimes. This intermittency is isolated to the fringes of the higher branch regime, at $\alpha=22.5^{\circ}$ and at $\alpha=7.5^{\circ}$ (these are highlighted in the striped region (red online) on the map in figure 8). Figure 13 shows the CWT of the oscillation measurements for increasing velocities at $\alpha=7.5^{\circ}$. As the velocity is increased the oscillations experience distinct aperiodic bursts with shorter regions of periodic behaviour. The analysed bandwidth was broader range than shown here but no significant spectral energy contribution was found. The contours at $U^{*}=7.9$ show a highly periodic signal with two distinct frequency bands with peaks at $f^{*}=0.7131$ and its harmonic $f^{*}=1.4262$, and occasional loss of periodicity. As the velocity is increased, the loss of periodicity is more prominent, with no discernible frequency and the cylinder barely oscillates.

The bursts of aperiodic behaviour observed here are in-line with the characteristics noted in intermittent transitions (Pomeau \& Manneville 1980), although whether it is mode competition or a route to chaos is unclear. Current existing tools used in analysis for these transitions are restricted to small degree of freedom dynamical systems, and inapplicable to the current observations. The identification of this intermittency paths are here resolved at low resolution, and expressed here as a function of $U^{*}$ for a fixed angle of attack. It is expected that at a higher parameter resolution, clearer relationships between the intermittency and the system variables could be elucidated.

\section{Conclusions}

This study investigated a square cylinder freely vibrating transverse to the flow. Although well known to gallop at zero angle of attack, we have shown that the diamond configuration exhibits an amplitude and frequency response comparable with the VIV experienced by circular cylinders. The results also reveal the influence of the shear layer separation on the fluid driving force experienced by the body, indicated by the diamond section locking to a Strouhal shedding frequency and maintaining the lower branch amplitude of oscillations to high velocities, in contrast to the circular cylinder. 

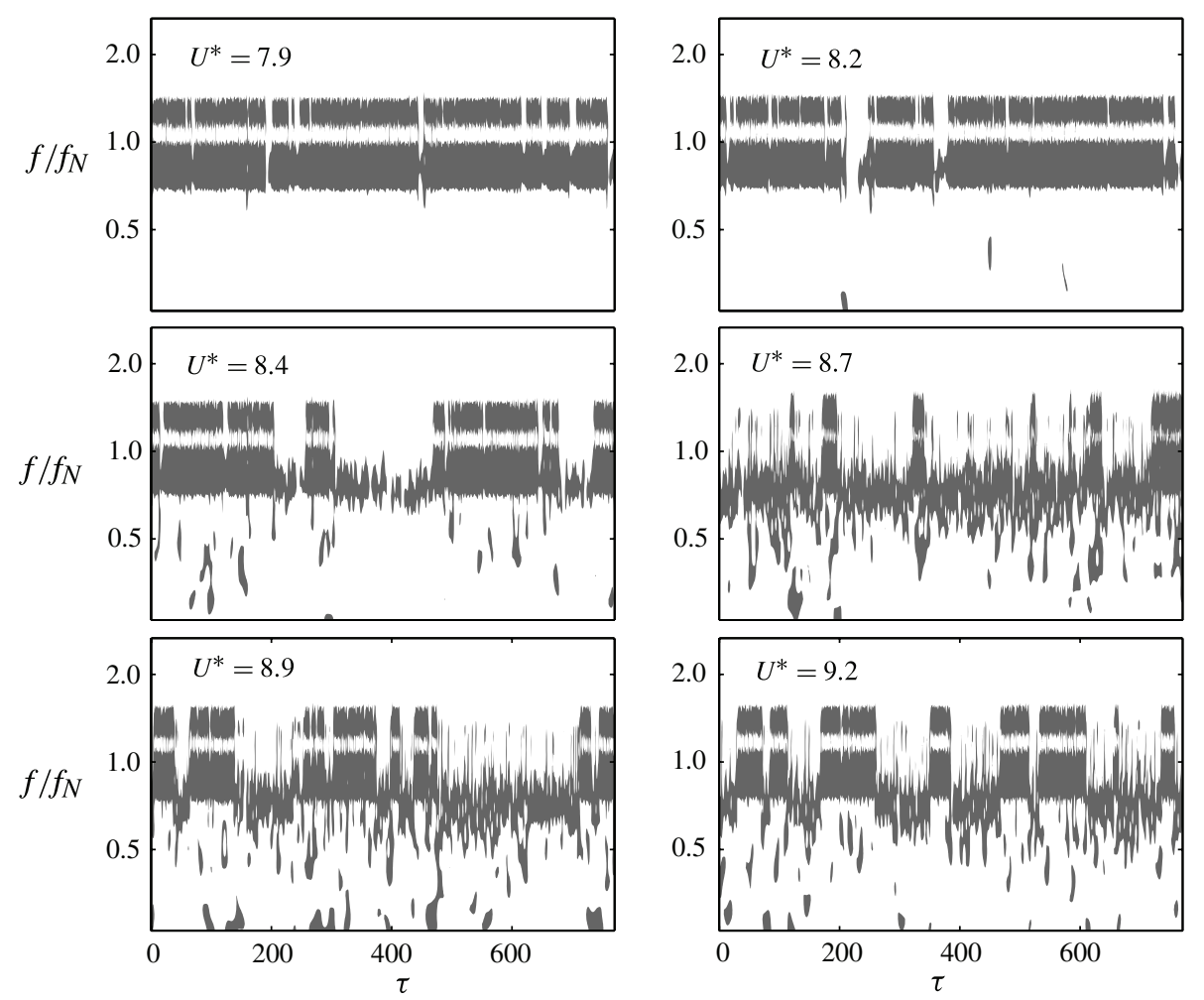

FIGURE 13. Normalized time history, $\tau=t f_{N}$, of energy contour plots of frequency at $\alpha=7.5^{\circ}$. The gaps in the contours highlight regions where the cylinder's periodic oscillation is disrupted. At $U^{*}=8.2$ the oscillations abruptly cease around $\tau=200-225$, while at higher velocities the oscillations with two frequencies are disrupted for durations up to 5 min $(\tau=190)$ with highly non-periodic motion.

By varying the angle of attack, we have shown the transition between the two phenomena to be a complex nonlinear combination of the modes, and uncovered a new branch of resonance. This previously unreported branch is characterized by an amplitude of oscillations at a frequency close to the subharmonic of the stationary shedding frequency and with an oscillation envelope in excess of amplitudes produced by either VIV or galloping over the reduced velocity range. Current oscillator models do not account for this new branch of oscillations, nor does quasi-steady theory. Current models will under predict the maximum amplitude and periodic loading on square cylinders when the angle of incidence varies.

Also confirmed is that the characteristic response of the galloping instability for a square cylinder is limited to angles under $\alpha=10^{\circ}$ for mass-damping ratio $m^{*} \zeta=6.49 \times 10^{-3}$. This has been confirmed over a wide range of reduced velocities. Outside this region the asymmetric body loading results in smaller oscillations offset from the centreline.

The results provide an experimental parameter map that can serve as a validation case for future studies. The work could be extended by investigating the nature of the geometry's influence and the universality of current FIV models, with a view to applying them to a wider range of body geometries, such as elliptic cylinders. 


\section{Acknowledgement}

This work was supported by the Australian Research Council through ARC Discovery Project Grant Number DP0774525.

\section{REFERENCES}

Assi, G. R. S., Meneghini, J. R., Aranha, J. A. P., Bearman, P. W. \& Casaprima, E. 2006 Experimental investigation of flow-induced vibration interference between two circular cylinders. J. Fluids Struct. 22, 819-827.

Bearman, P. W. 1984 Vortex shedding from oscillating bluff bodies. Annu. Rev. Fluid Mech. 16, 195-222.

Bearman, P. W., Gartshore, I. S., Maull, D. J. \& Parkinson, G. V. 1987 Experiments on flow-induced vibration of a square-section cylinder. J. Fluids Struct. 1, 19-34.

Blevins, R. D. (Ed.) 1990 Flow-induced Vibration. Von Nostrand Reinhold.

Bokaian, A. R. \& Geoola, F. 1983 On the cross flow response of cylindrical structures. Proc. Inst. Cir. Engng 75, 397-418.

Bokaian, A. R. \& Geoola, F. 1984 Hydroelastic instabilities of square cylinders. J. Sound Vib. 92, 117-141.

Bouclin, D. N. 1977 Hydroelastic oscillations of square cylinders. Master's thesis, University of British Columbia, Vancouver, BC, Canada.

Carberry, J., Govardhan, R., Sheridan, J., Rockwell, D. \& Williamson, C. H. K. 2004 Wake states and response branches of forced and freely oscillating cylinders. Eur. J. Mech. 23, 89-97.

Corless, R. M. \& Parkinson, G. V. 1988 A model of the combined effects of vortex-induced oscillation and galloping. J. Fluids Struct. 2, 203-220.

Corless, R. M. \& PARKinson, G. V. 1993 Mathematical modelling of the combined effects of vortex-induced vibration and galloping. Part II. J. Fluids Struct. 7, 825-848.

Den Hartog, J. P. 1932 Transmission line vibration due to sleet. Trans. AIEE 51, 1074-1076.

Deniz, S. \& StAubli, T. 1997 Oscillating rectangular and octagonal profiles: interaction of leadingand trailing-edge vortex formation. J. Fluids Struct. 11, 3-32.

Dutta, S., Panigrahi, P. K. \& Muralidhar, K. 2008 Experimental investigation of flow past a square cylinder at an angle of incidence. J. Engng Mech. 134, 788-803.

FENG, C. C. 1968 The measurement of vortex-induced effects in flow past stationary and oscillating circular and D-section cylinders. Master's thesis, University of British Columbia, Vancouver, BC, Canada.

Fouras, A., Lo Jacono, D. \& Hourigan, K. 2008 Target-free stereo PIV: a novel technique with inherent error estimation and improved accuracy. Exp. Fluids 44, 317-329.

Govardhan, R. \& Williamson, C. H. K. 2000 Modes of vortex formation and frequency response of a freely vibrating cylinder. J. Fluid Mech. 420, 85-130.

Govardhan, R. \& Williamson, C. H. K. 2004 Critical mass in vortex-induced vibration of a cylinder. Eur. J. Mech. 23, 17-27.

Govardhan, R. N. \& Williamson, C. H. K. 2006 Defining the 'modified Griffin plot' in vortex-induced vibration: revealing the effect of Reynolds number using controlled damping. J. Fluid Mech. 561, 147-180.

Hover, F. S., Techet, A. H. \& Triantafyllou, M. S. 1998 Forces on oscillating uniform and tapered cylinders in cross flow. J. Fluid Mech. 363, 97-114.

KhalaK, A. \& Williamson, C. H. K. 1996 Dynamics of a hydroelastic cylinder with very low mass and damping. J. Fluids Struct. 10, 455-472.

Khalak, A. \& Williamson, C. H. K. $1997 a$ Fluid forces and dynamics of a hydroelastic structure with very low mass and damping. J. Fluids Struct. 11, 973-982.

KHAlak, A. \& Williamson, C. H. K. $1997 b$ Investigation of relative effects of mass and damping in vortex-induced vibration of a circular cylinder. J. Wind Engng. Ind. Aerodyn. 69-71, 341-350. 
Khalak, A. \& Williamson, C. H. K. 1999 Motions, forces and mode transitions in vortex-induced vibrations at low mass-damping. J. Fluids Struct. 13, 813-851.

Klamo, J. T., Leonard, A. \& Roshko, A. 2006 The effects of damping on the amplitude and frequency response of a freely vibrating cylinder in cross-flow. J. Fluids Struct. 22, 845-856.

Leontini, J. S., Stewart, B. E., Thompson, M. C. \& Hourigan, K. 2006 Wake state and energy transitions of an oscillating cylinder at low Reynolds number. Phys. Fluids 18, 067101.

Luo, S. C., Tong, X. H. \& Khoo, B. C. 2007 Transition phenomena in the wake of a square cylinder. J. Fluids Struct. 23, 227-248.

Morse, T. L., Govardhan, R. N. \& Williamson, C. H. K. 2008 The effect of end conditions on the vortex-induced vibration of cylinders. J. Fluids Struct. 24, 1227-1239.

Naudascher, E. \& Rockwell, D. 1994 Flow-induced Vibrations: an Engineering Guide. A. A. Balkema.

NAUdASCHER, E. \& WANG, Y. 1993 Flow-induced vibrations of prismatic bodies and grids of prisms. J. Fluids Struct. 7, 341-373.

NoRBERG, C. 1993 Flow around rectangular cylinders: pressure forces and wake frequencies. J. Wind Engng. Ind. Aerodyn. 49, 187-196.

Obasaju, E. D., Ermshaus, R. \& Naudascher, E. 1990 Vortex-induced streamwise oscillations of a square-section cylinder in a uniform stream. J. Fluid Mech. 213, 171-189.

OKAJima, A. 1982 Strouhal numbers of rectangular cylinders. J. Fluid Mech. 123, 379-398.

PARKInSON, G. 1989 Phenomena and modelling of flow-induced vibrations on bluff bodies. Prog. Aerosp. Sci. 26, 169-224.

PARKinson, G. V. \& SMith, J. D. 1964 The square prism as an aeroelastic nonlinear oscillator. Q. J. Mech. Appl. Maths 17, 225-239.

PARKINSON, G. V. \& WAWZONEK, M. A. 1981 Some considerations of combined effects of galloping and vortex resonance. J. Wind Engng. Ind. Aerodyn. 8, 135-143.

Pomeau, Y. \& Manneville, P. 1980 Intermitten transition to turbulence in dissipative dynamical systems. Commun. Math. Phys. 74, 189-197.

SARPKAYA, T. 1979 Vortex-induced oscillations: a selective review. J. Appl. Mech. 46, 241-258.

SARPKAYA, T. 2004 A critical review of the intrinsic nature of vortex-induced vibrations. J. Fluids Struct. 19, 389-447.

Sheard, G. J., Fitzgerald, M. J. \& Ryan, K. 2009 Cylinders with square cross-section: wake instabilities with incidence angle variation. J. Fluid Mech. 630, 43-69.

Tong, X. H., Luo, S. C. \& Khoo, B. C. 2008 Transition phenomena in the wake of an inclined square cylinder. J. Fluids Struct. 24, 994-1005.

van Oudheusden, B. W., Scarano, F., van Hinsberg, N. P. \& Roosenboom, E. W. M. 2008 Quantitative visualization of the flow around a square-section cylinder at incidence. J. Wind Engng Ind. Aerodyn. 96, 913-922.

VICKERY, B. J. 1966 Fluctuating lift and drag on a long cylinder of square cross-section in a smooth and in a turbulent stream. J. Fluid Mech. 25, 481-494.

WANG, Z. J. \& ZHOU, Y. 2005 Vortex-induced vibration characteristics of an elastic square cylinder on fixed supports. J. Fluids Engng 127, 241-249.

Williamson, C. H. K. \& Govardhan, R. N. 2004 Vortex-induced vibrations. Annu. Rev. Fluid Mech. 36, 413-455.

Williamson, C. H. K. \& RoshKo, A. 1988 Vortex formation in the wake of an oscillating cylinder. J. Fluids Struct. 2, 355-381.

Yoon, D., YANG, K. \& CHOI, C. 2010 Flow past a square cylinder with an angle of incidence. Phys. Fluids 22, 043603. 